\title{
15. PALEOENVIRONMENTAL VARIATION BASED ON THE MINERALOGY AND ROCK-MAGNETIC PROPERTIES OF SEDIMENT FROM SITES 885 AND 886
}

\author{
Eve Arnold, ${ }^{2}$ Margaret Leinen, ${ }^{2}$ and John King ${ }^{2}$
}

\begin{abstract}
Variation in the mineralogy and rock magnetic properties of Sites 885 and 886 sediment can be explained by changing environmental conditions in the sediment source areas and sediment column. Climatic variations produce changes in the mineralogy and magnetics at these sites consistent with aridification of the Asian source area and cooling forced by tectonic activity. Asian aridification is noted by gradual reduction of a kaolinite-rich mineral assemblage from the late Miocene to an abrupt mineralogy change at 3.8 Ma to a chlorite- and illite-rich mineral assemblage. At the same time, the eolian mass accumulation rate and ferrimagnetic grain size increases. This event precedes the onset of Asian loess deposition by over $1 \mathrm{~m} . \mathrm{y}$. , but it is consistent with a coupled environmental mechanism that explains the rapid onset of loess sedimentation at $2.5 \mathrm{Ma}$. Both the mineralogy and rock magnetics are overprinted with a diagenetic signal that suggests the sediments proximal to Sites 885 and 886 may have been suboxic to anoxic in the early Pliocene.
\end{abstract}

\section{INTRODUCTION}

The pelagic sediments preserved near Ocean Drilling Program (ODP) Holes $885 \mathrm{~A}$ and $886 \mathrm{~B}$ in the North Pacific Ocean are largely eolian in origin (Griffin et al., 1968; Leinen, 1989; Kyte et al., 1993); thus, variations in the properties of these sediments are related to variations in atmospheric transport patterns. The present upwind source region for these eolian sediments is Asia, and the sediments record both past climatic variation and tectonic activity from this continent. In addition, the terrigenous material may have been subjected to sediment reworking and diagenetic alteration. By examining a variety of parameters, we can attempt to deconvolve this climate-tectonictransport-preservation signal into its individual components and learn something about the variation of each through time.

In this paper, we examine the downcore variation in mineralogy and rock-magnetic measurements to infer the paleoenvironmental conditions responsible for sedimentation proximal to Sites 885 and 886 . The composition and concentration of terrigenous material preserved in deep-sea sediment are related to weathering, transport, and depositional processes as well as postdepositional alteration of continental material. Mineralogical composition is related to parentrock composition as well as regional climate. Paleoclimatologists and atmospheric scientists have established that the mineralogy of present-day aerosols is related to the source area (M. Leinen et al., unpubl. data; J. Merrill et al., unpubl. data) and that the mineralogy of aerosols collected over the North Pacific is the same as that of surface sediments from eolian deposits (Blank et al., 1985). Furthermore, downcore variation in mineralogy has demonstrated a consistent relationship with other paleoclimate proxies and has allowed paleoclimatologists to interpret variation in past climates in the Asia-North Pacific region (Leinen, 1985, 1989; Schramm, 1989).

In an analogous manner, rock-magnetic studies have also been used to trace atmospheric samples to their source area (Oldfield et al., 1985 ) and to infer changes in atmospheric input to deep-sea sediments (Robinson, 1986; Doh et al., 1988). In addition to compositional information, rock-magnetic properties are also useful proxies for the concentration and grain size of terrigenous deep-sea sediments. Finally, the rock-magnetic properties are very sensitive to

\footnotetext{
${ }^{1}$ Rea, D.K., Basov, I.A., Scholl, D.W., and Allan, J.F. (Eds.), 1995. Proc. ODP, Sci. Results, 145: College Station, TX (Ocean Drilling Program).

${ }^{2}$ University of Rhode Island, Graduate School of Oceanography, Narragansett, RI 02882, U.S.A.
}

reduction diagenesis, so that we can control for postdepositional alteration of the sediment.

The rock-magnetic analyses are nondestructive, so we were able to analyze the same samples for mineralogy. Because the mineralogy of atmospheric aerosols has been identified with broad regional source areas, we can use this information to interpret the mineralogy signal in deep-sea sediments if we can confirm that the original mineralogy has not been modified by transport, deposition, or postdepositional modification. Bulk mineralogy can be altered by transport and deposition because mineralogy varies with grain size. As material travels through the atmosphere and falls through the water column, large particles fall out more quickly than the fine fraction. In addition, winnowing of deep-sea sediments can result in size fractionation. The coarse fraction of terrigenous material is relatively concentrated in primary minerals such as quartz and plagioclase, whereas the clay minerals dominate the fine fraction. We control for this by examining the mineralogy in separate size classes in the deepsea sediments. Diagenetic alteration may be discerned by comparing the mineralogy record with the rock-magnetic properties; divergence of these two signals may indicate either a postdepositional modification of the sediment or a change in source.

\section{METHODS}

Samples were collected at $150 \mathrm{~cm}$ intervals from Holes $885 \mathrm{~A}$ and $886 \mathrm{~B}$ with an aluminum sampling tool designed to collect undisturbed sediment; they were then extruded into $5-\mathrm{cm}^{3}$ plastic cubes for rock-magnetic analyses. In addition, ODP paleomagnetic samples (7 $\mathrm{cm}^{3}$ ), collected at $150 \mathrm{~cm}$ intervals, were also used. Sample spacing for the rock-magnetic study is approximately $75 \mathrm{~cm}$, and $150 \mathrm{~cm}$ for the mineralogical analyses.

\section{Rock Magnetics}

Susceptibility $\left(X_{l f}, X_{h f}\right)$ was measured on a Bartington Instruments susceptibility meter at 0.47 and $4.7 \mathrm{kHz}$. The reported value (in $10^{-6} \mathrm{~m}^{3} / \mathrm{g}$ ) is the average of three replicates. The samples were demagnetized in a $100 \mathrm{mT}$ alternating field. Anhysteretic remanent magnetization $(A R M)$ was induced in a $0.1 \mathrm{mT}$ steady field superimposed on a $100 \mathrm{mT}$ alternating field. The $A R M$ was measured on a cryogenic magnetometer, and the reported value (in $10^{-6} \mathrm{Am}^{2} / \mathrm{g}$ ) is the average of duplicate measurements. The $X_{a r m}$ was calculated by dividing $A R M$ by the steady field (reported in $10^{-6} \mathrm{~m}^{3} / \mathrm{g}$ ). The final set of magnetic measurements were saturated isothermal remanent magnetization (SIRM) 
and isothermal remanent magnetization $\left(I R M_{-0.3 T}\right)$. Samples were saturated in a $1.2 \mathrm{~T}$ field and measured on a cryogenic magnetometer. Samples were then placed in reverse fields of $0.3 \mathrm{~T}$ and the magnetization remeasured. The values reported (in $10^{-6} \mathrm{Am}^{2} / \mathrm{g}$ ) are averages of duplicate measurements. The frequency ratio $\left(X_{h f} / X_{l f}\right)$, the grain size parameter $\left(X_{a r m} / X_{l f}\right)$, and the compositional parameters $(S[S=$ $\left.I R M_{-0.3 T} / S I R M\right]$ and HIRM $\left.\left[H I R M=\left\{I R M_{-0.3 T}+S I R M\right\} / 2\right]\right)$ were calculated from the primary measurements. Further explanation of these variables and their applications may be found in the "Results" section below. Readers unfamiliar with rock-magnetic techniques are referred to King et al. (1989).

\section{Mineralogy}

Samples were freeze-dried, weighed, and wet sieved at $63 \mu \mathrm{m}$; the $>63 \mu \mathrm{m}$ and $<63 \mu \mathrm{m}$ were then dried and weighed. The $<63 \mu \mathrm{m}$ fraction was treated to remove biogenic silica using a $\mathrm{NaOH}$ procedure (see Snoeckx et al., this volume). The $\mathrm{NaOH}$ procedure removes more amorphous material than other common extraction techniques, but does not alter the relative proportion of the various mineral phases. Iron oxides were removed using the oxalic acid extraction technique of Landa and Gast (1973). Sediments were saturated with $\mathrm{MgCl}_{2}$, to reduce $d$-spacing variability caused by cation differences; they were then rinsed with warm deionized water, dried, and weighed. Sediments were wet sieved at $20 \mu \mathrm{m}$ with the aid of a sonic dismembrator, and the $<20 \mu \mathrm{m}$ fraction was split into $2-20 \mu \mathrm{m}$ and $<2 \mu \mathrm{m}$ size fractions by means of centrifugation. The $2-20 \mu \mathrm{m}$ size fraction was spiked with a $10 \%$ by weight $\mathrm{Al}_{2} \mathrm{O}_{3}$ internal standard and the $<2 \mu \mathrm{m}$ fraction was spiked with a $10 \%$ talc internal standard. Samples were homogenized by grinding in a mortar and pestle under acetone, then air dried, suspended in a deionized water slurry, and drawn onto duplicate (one air dried, one glycolated) silver filters for X-ray analysis.

The X-ray analysis was run from $2^{\circ}$ to $30^{\circ} 2 \theta$ at $45 \mathrm{kV}$ and $40 \mathrm{~mA}$ at $2^{\circ} 2 \theta / \mathrm{min}$ using $\mathrm{CuK} \alpha$ radiation. Peak areas for smectite, illite, kaolinite, chlorite, quartz, and plagioclase were determined using Scintag DMS software. Precision of the peak areas is listed in Table 1. These precisions were calculated by comparing the area under the mineral curves between the glycolated and unglycolated samples. Smectite cannot be compared in this manner. Replicate scans and peak area resolution for this mineral were performed; the error for smectite determination is high (in excess of $\sim 30 \%$ for both size fractions). The relative proportion of kaolinite and chlorite was determined by the relative proportions of the kaolinite [002] and chlorite [004] peak areas. Mineral peak areas were normalized to the internal standard peak areas.

\section{Stratigraphy, Correlation, Age Model, and Mass Accumulation Rates}

Sites $885\left(44^{\circ} 41^{\prime} \mathrm{N}, 168^{\circ} 16^{\prime} \mathrm{E}\right)$ and $886\left(44^{\circ} 41^{\prime} \mathrm{N}, 168^{\circ} 14^{\prime} \mathrm{E}\right)$ were drilled at about $5700 \mathrm{~m}$ water depth in the North Pacific red-clay province. A total of $59 \mathrm{~m}$ of sediments were recovered from Hole $885 \mathrm{~A}$ and $69 \mathrm{~m}$ from Hole $886 \mathrm{~B}$. Three stratigraphic sedimentary units were recognized at each site: (I) Pleistocene to late Pliocene reddish brown to brown red clay; (II) late Pliocene to late Miocene diatom ooze, and (III) a lower brown clay unit, originally dated as late Miocene (Rea, Basov, Janecek, Palmer-Julson, et al., 1993), but now recognized as older; late Miocene to late Cretaceous in Hole $886 \mathrm{C}$ (Ingram, this volume).

Table 2 lists the stratigraphic parameters for Holes $885 \mathrm{~A}$ and $886 \mathrm{~B}$. Correlation between and composite depth of Holes $885 \mathrm{~A}$ and $886 \mathrm{~B}$ is detailed in Dickens et al. (this volume); all depths in this report are composite depths derived from that model. The depth intervals (in meters composite depth [mcd]) for Unit I are 0-23.5 med; for Unit II, 23.5 to $\sim 52 \mathrm{mcd}$, and for Unit III, $>52 \mathrm{mcd}$. The age models used in this report are derived from the magnetic reversal stratigraphy listed in Dickens et al. (this volume). These ages are in agreement with the Radiolaria stratigraphy reported by Morely (this volume). Ages derived from ichthyolith strontium isotopic ratios (Ingram, this volume) indicate slightly younger sediments in the lower Unit II samples, but older sediment in the Unit III sediments than in the magnetic age model. Hiatuses and low sedimentation rates explain the discrepancy between the Unit III ages. Because the radiolarian ages agree with the paleomagnetic estimates, the $\mathrm{Sr}$ data were not incorporated into the age model for this study.

The sedimentation rate is estimated by linear interpolation between each magnetic datum obtained from the composite depth model. It is important to recognize that the linear sedimentation rates (LSRs) derived from the composite depth section may be higher than those derived from the original cores. This discrepancy is cause for some concern when trying to estimate mass accumulation rates (MARs), as the seismic records indicate that the Site 885 sediment is indeed thinner than Site 886 (Rea, Basov, Janecek, Palmer-Julson, et al., 1993). Most of the difference appears to occur in the lower brown clay of Unit III. The LSRs derived from the composite depth model are comparable with those derived from the original depths from Hole 886B for all stratigraphic units. However, the LSR for Hole 885A Unit I is lower $(0.48 \mathrm{~cm} / \mathrm{k} . \mathrm{y}$.) than the Unit I LSR estimated from the composite depth model $(0.67 \mathrm{~cm} / \mathrm{k}$.y.). A comparison of depth intervals between susceptibility datum for Holes $885 \mathrm{~A}$ and $886 \mathrm{~A}$ indicates equal LSRs in the top $10 \mathrm{~m}$ of these cores. Thus, it appears reasonable to apply the same LSRs to both sites for the upper two units, because the difference in the LSR estimates may be explained by coring gaps or drilling disturbance.

Dry bulk density (DBD) was estimated for each sample based on the dry weights of the constant volume magnetic cubes. The percent terrigenous is operationally defined as the $<63 \mu \mathrm{m}$ material remaining after the biogenic silica and iron oxide removals, expressed as a weight percent of the total original sample. The $2-20 \mu \mathrm{m}$ and $<2 \mu \mathrm{m}$ percentages are the weight percents of the total original sample for each size class after chemical treatment. The bulk mass accumulation rate (MAR) is the product of the LSR and DBD. The terrigenous, $2-20 \mu \mathrm{m}$, and $<2 \mu \mathrm{m}$ MARs are the product of the bulk MAR and the respective weight percent for each sedimentary component. The accumulation rates for the rock-magnetic properties are the product of the rock magnetic measurement and the bulk MAR. The accumulation of the $<2 \mu \mathrm{m}$ minerals is the product of the normalized peak area and the $<2 \mu \mathrm{m}$ MAR.

\section{RESULTS \\ Rock Magnetics}

Figure 1 illustrates the rock magnetic parameters plotted vs. composite depth, and Table 3 lists the magnetic measurements for Holes $885 \mathrm{~A}$ and $886 \mathrm{~B}$. The low-frequency susceptibility (Fig. 1A) is primarily a measure of the concentration of magnetic iron oxides and is used as a proxy for the concentration of terrigenous material. Susceptibility is highest in stratigraphic Unit III, the lower brown clay unit, suggesting a large iron oxide component in these sediments, which grades monotonically to low values in the lower part of Unit II, the diatom ooze ( $\sim 6 \mathrm{mcd})$. Susceptibility remains low throughout the diatom ooze and increases at the base of Unit I, the upper red clay unit ( $24 \mathrm{mcd})$. There are large-amplitude peaks superimposed on the general upcore increase in susceptibility, coincident with the ash layers described in the Leg 145 Initial Reports (Rea, Basov, Janecek, Palmer-Julson, et al., 1993).

The frequency dependence of the susceptibility $\left(X_{h j} / X_{i j}\right)$ is used to identify the presence of very fine (submicrometer), viscous, superparamagnetic grains. Should the contribution of such grains be large (low $X_{h f} / X_{l f}$ ratios), the susceptibility concentration proxies and grainsize parameters derived from these values will no longer be related to the concentration and grain size of terrigenous materials, as superparamagnetic materials make a disproportionately large contribution to the susceptibility. Figure 1B illustrates the frequency dependence of the susceptibility; the frequency dependence in the lower and upper 

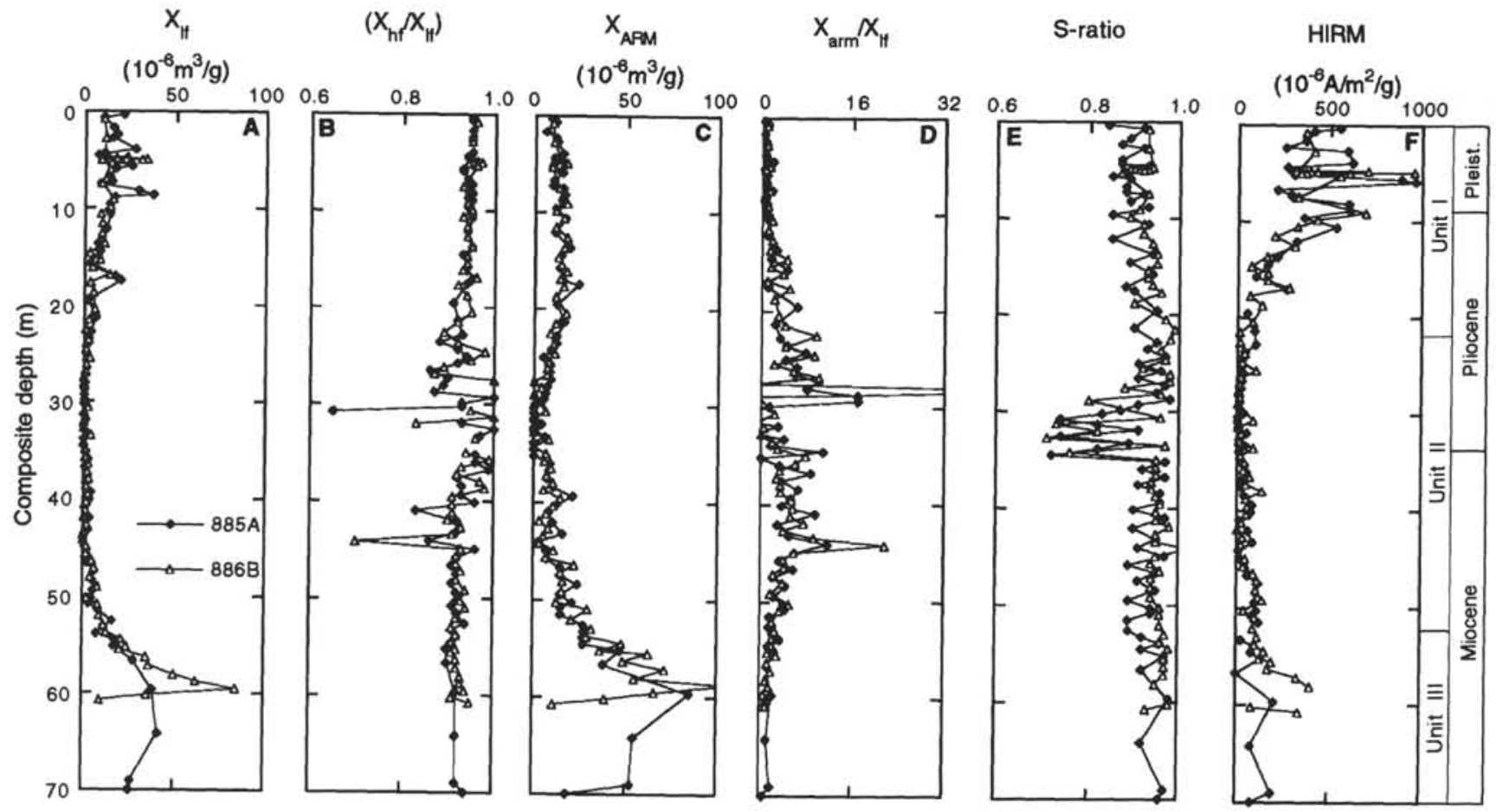

Figure 1. Rock-magnetic parameters vs. composite depth for Holes 885A and 886B.

red clay units is relatively uniform and low, indicating that the application of the magnetic proxies for terrigenous material is appropriate in these intervals. The diatom ooze unit displays a more variable frequency dependence signal, largely because of the low concentration of terrigenous material combined with a negative contribution of the biogenic silica to the susceptibility signal, resulting in a frequency ratio of two very small numbers. However, there does not appear to be a large viscous superparamagnetic contribution in this stratigraphic unit; again, the application of the magnetic parameters as terrigenous proxies is appropriate.

Anhysteretic remanent magnetization, expressed as $X_{\text {arm }}$ (Fig. 1C), is another measure of iron oxide concentration, but it is also affected by the domain state of the magnetic minerals. The domain state of magnetic material is related to the iron oxide particle grain size, which, in turn, may be related to the terrigenous grain size, if fine magnetic particles are not randomly incorporated into larger terrigenous grains. The iron oxide grain size is related to the terrigenous grain size because magnetic particles such as magnetite and hematite, derived from continental source areas, are subject to the same transport processes as the alumino-silicate grains. Smaller magnetic grains yield a larger $X_{a r m}$ signal. The $X_{a r m}$ mimics the upcore pattern observed for the susceptibility in the lower clay and diatom units, but reaches a broad maximum between 10 and $20 \mathrm{mcd}$ in Unit I before decreasing toward the top of the core. This decrease at the top of the core indicates either a decrease in concentration of magnetic material, in conflict with the susceptibility signal, or an increase in the magnetic grain size. Examination of the ratio $X_{a r m} / X_{i f}$ (Fig. 1D) indicates that the latter explanation is more likely.

The ratio $X_{a r m} / X_{l f}$ is inversely related to the ferrimagnetic iron oxide grain size and is independent of magnetic concentration. The $X_{a r m} / X_{l f}$ ratio monotonically increases from the base of stratigraphic Unit III to the base of stratigraphic Unit II, indicating a general ferrimagnetic-iron oxide grain-size decrease throughout the lower brown clay unit. The ratio abruptly increases and becomes more variable at the base of Unit II. The high variability in this unit is related to the low magnetic mineral concentration in the diatom ooze; however, a few comments may be made about the overall grain-size pattern. In general, the diatom ooze contains the finest grain size of all
Table 1. Precision, expressed as percent relative error of mineral peak areas.

\begin{tabular}{rcccccc}
\hline Size & Smectite & Illite & Kaolinite & Chlorite & Quartz & Plagioclase \\
\hline$<2 \mu \mathrm{m}$ & ND & 2.3 & 11.2 & 2.9 & 7.6 & 2.2 \\
$2-20 \mu \mathrm{m}$ & ND & 1.3 & 33.3 & 0.4 & 3.0 & 2.5 \\
\hline
\end{tabular}

Note: $\mathrm{ND}=$ not determined.

sediments from Holes $885 \mathrm{~A}$ and $886 \mathrm{~B}$. There is an interval of low $X_{a r m} / X_{i f}$ between 34 and $30 \mathrm{mcd}$, indicating a relatively coarse mag. netic grain size, and a sharp peak in the grain-size ratio at $\sim 28-30$ mcd, indicating a very fine magnetic grain size. Above $\sim 28 \mathrm{mcd}$, the grainsize ratio decreases and becomes less variable throughout the remainder of the diatom ooze and in all of stratigraphic Unit I. This pattern of variation suggests a general increase in the ferrimagnetic grain size toward the top of the core.

The S-ratio $\left(I R M_{-0.3 T} / S I R M_{1,2 \mathrm{~T}}\right)$ is a parameter sensitive to the composition of magnetic minerals. Magnetic minerals such as magnetite are easily magnetized, whereas minerals such as hematite require stronger magnetic fields to saturate them. Thus, the ratio of the proportion of magnetization stripped off of the magnetically saturated sample in a reverse field of $0.3 \mathrm{~T}$ is related to the proportion of "hard" to "soft" magnetic material present in a sample. Figure IE shows the S-ratio for Sites 885 and 886 . The small-amplitude, high-frequency variability in Hole $885 \mathrm{~A}$ sediments is a systematic laboratory error; one batch of samples was not completely saturated. The S-ratio is relatively uniform and high in the lower brown clay unit and in the lower part of the diatom unit, indicating a constant magnetic mineral composition throughout this interval. Low S-ratios are observed from 29 to $35 \mathrm{mcd}$; this is the same interval as the low $X_{a r m} / X_{l f}$ ratios described earlier. The remainder of the diatom ooze unit and the upper red clay unit indicates a gradual evolution toward a slightly harder coercivity toward the top of the core.

HIRM ([IRM ${ }_{-0.3 T}+$ SIRM $] / 2$; Fig. 1F) is used to estimate the concentration of hard magnetic material in the sediment. HIRM occurs at moderate concentrations in the lower brown clay unit and 
Table 2. Stratigraphic parameters and mass accumulation rates for Holes $885 \mathrm{~A}$ and $\mathbf{8 8 6 \mathrm { B }}$.

\begin{tabular}{|c|c|c|c|c|c|c|c|c|c|c|c|}
\hline $\begin{array}{l}\text { Core, section, } \\
\text { interval }(\mathrm{cm})\end{array}$ & $\begin{array}{l}\text { Depth } \\
\text { (mbsf) }\end{array}$ & $\begin{array}{l}\text { Composite } \\
\text { depth } \\
\text { (m) }\end{array}$ & $\begin{array}{l}\text { Age } \\
(\mathrm{Ma})\end{array}$ & $\begin{array}{c}\text { LSR } \\
(\mathrm{cm} / \mathrm{k} . \mathrm{y} .)\end{array}$ & $\begin{array}{l}\text { Dry bulk } \\
\text { density } \\
\left(\mathrm{g} / \mathrm{cm}^{3}\right)\end{array}$ & $\begin{array}{c}\text { Bulk } \\
\text { MAR } \\
\left(\mathrm{g} / \mathrm{cm}^{2} / \mathrm{k} . \mathrm{y} .\right)\end{array}$ & $\begin{array}{c}\text { Terrigenous } \\
\text { MAR } \\
\text { (g/cm²/k.y.) }\end{array}$ & $\begin{array}{c}>63 \mu \mathrm{m} \\
\mathrm{MAR} \\
\left(\mathrm{g} / \mathrm{cm}^{2} / \mathrm{k} . \mathrm{y} .\right)\end{array}$ & $\begin{array}{c}20-63 \mu \mathrm{m} \\
\mathrm{MAR} \\
\left(\mathrm{g} / \mathrm{cm}^{2} / \mathrm{k} . \mathrm{y} .\right)\end{array}$ & $\begin{array}{c}2-20 \mu \mathrm{m} \\
\mathrm{MAR} \\
\left(\mathrm{g} / \mathrm{cm}^{2} / \mathrm{k} . \mathrm{y} .\right)\end{array}$ & $\begin{array}{c}<2 \mu \mathrm{m} \\
\mathrm{MAR} \\
\left(\mathrm{g} / \mathrm{cm}^{2} \mathrm{k} \cdot \mathrm{y} .\right)\end{array}$ \\
\hline \multicolumn{12}{|l|}{$145-885 \mathrm{~A}-$} \\
\hline $1 \mathrm{H}-1,25$ & 0.25 & 0.30 & 0.05 & 0.63 & 0.47 & 0.295 & 0.143 & 0.014 & 0.003 & 0.092 & 0.042 \\
\hline $1 \mathrm{H}-1,125$ & 1.25 & 1.65 & 0.26 & 0.63 & 0.54 & 0.337 & 0.152 & 0.010 & 0.003 & 0.097 & 0.045 \\
\hline $1 \mathrm{H}-2,25$ & 1.75 & 2.34 & 0.37 & 0.63 & 0.45 & 0.284 & 0.144 & 0.007 & 0.004 & 0.094 & 0.043 \\
\hline $1 \mathrm{H}-2,125$ & 2.75 & 3.89 & 0.62 & 0.63 & 0.44 & 0.277 & 0.127 & 0.009 & 0.008 & 0.081 & 0.033 \\
\hline $1 \mathrm{H}-3,25$ & 3.25 & 4.34 & 0.69 & 0.63 & 0.47 & 0.291 & 0.129 & 0.006 & 0.002 & 0.080 & 0.042 \\
\hline $1 \mathrm{H}-3,125$ & 4.25 & 5.16 & 0.85 & 0.41 & 0.48 & 0.194 & 0.103 & 0.002 & 0.003 & 0.066 & 0.031 \\
\hline $2 \mathrm{H}-1,25$ & 4.85 & 5.63 & 0.96 & 0.41 & 0.53 & 0.217 & 0.118 & 0.002 & 0.004 & 0.075 & 0.036 \\
\hline $2 \mathrm{H}-1,125$ & 5.85 & 6.72 & 1.11 & 0.46 & 0.47 & 0.216 & 0.099 & 0.004 & 0.004 & 0.066 & 0.025 \\
\hline $2 \mathrm{H}-2,25$ & 6.35 & 7.24 & 1.27 & 0.46 & 0.55 & 0.252 & 0.128 & 0.004 & 0.004 & 0.078 & 0.035 \\
\hline $2 \mathrm{H}-2,125$ & 7.35 & 8.19 & 1.48 & 0.46 & 0.47 & 0.217 & 0.092 & 0.008 & 0.006 & 0.057 & 0.023 \\
\hline $2 \mathrm{H}-3,25$ & 7.85 & 8.63 & 1.57 & 0.46 & 0.57 & 0.261 & 0.093 & 0.030 & 0.017 & 0.055 & 0.014 \\
\hline $2 \mathrm{H}-3,125$ & 8.85 & 9.61 & 1.78 & 0.46 & 0.44 & 0.205 & 0.083 & 0.004 & 0.003 & 0.052 & 0.023 \\
\hline $2 \mathrm{H}-4,125$ & 10.35 & 12.12 & 2.23 & 0.66 & 0.52 & 0.342 & 0.158 & 0.002 & 0.004 & 0.097 & 0.048 \\
\hline $2 \mathrm{H}-5,125$ & 11.85 & 14.55 & 2.60 & 0.66 & 0.44 & 0.290 & 0.116 & 0.002 & 0.002 & 0.072 & 0.035 \\
\hline $2 \mathrm{H}-6,125$ & 13.35 & 17.06 & 2.86 & 0.96 & 0.42 & 0.407 & 0.091 & 0.008 & 0.011 & 0.056 & 0.017 \\
\hline $3 \mathrm{H}-\mathrm{I}, 125$ & 15.35 & 21.35 & 3.30 & 0.96 & 0.48 & 0.458 & 0.132 & 0.005 & 0.006 & 0.079 & 0.038 \\
\hline $3 \mathrm{H}-2,125$ & 16.85 & 23.55 & 3.53 & 0.96 & 0.37 & 0.354 & 0.062 & 0.005 & 0.002 & 0.041 & 0.015 \\
\hline $3 \mathrm{H}-3,125$ & 18.35 & 25.05 & 3.77 & 0.61 & 0.33 & 0.202 & 0.015 & 0.005 & 0.001 & 0.010 & 0.003 \\
\hline $3 \mathrm{H}-4,125$ & 19.85 & 26.55 & 4.02 & 0.61 & 0.32 & 0.192 & 0.019 & 0.001 & 0.001 & 0.010 & 0.004 \\
\hline $3 \mathrm{H}-5,125$ & 21.35 & 28.05 & 4.26 & 0.61 & 0.28 & 0.171 & 0.012 & 0.001 & 0.001 & 0.008 & 0.003 \\
\hline $3 \mathrm{H}-6,125$ & 22.85 & 29.31 & 4.47 & 0.61 & 0.29 & 0.177 & 0.008 & 0.001 & 0.000 & 0.006 & 0.001 \\
\hline $4 \mathrm{H}-1,125$ & 24.85 & 30.82 & 4.72 & 0.61 & 0.24 & 0.143 & 0.004 & 0.001 & 0.000 & 0.002 & 0.001 \\
\hline $4 \mathrm{H}-2,125$ & 26.35 & 31.95 & 4.91 & 0.61 & 0.29 & 0.178 & 0.014 & 0.003 & 0.000 & 0.009 & 0.003 \\
\hline $4 \mathrm{H}-3,125$ & 27.85 & 33.23 & 5.12 & 0.61 & 0.40 & 0.241 & 0.054 & 0.002 & 0.002 & 0.034 & 0.015 \\
\hline $4 \mathrm{H}-4,125$ & 29.35 & 34.53 & 5.33 & 0.61 & 0.36 & 0.217 & 0.027 & 0.002 & 0.002 & 0.017 & 0.005 \\
\hline $4 \mathrm{H}-5,125$ & 30.85 & 35.99 & 5.57 & 0.61 & 0.39 & 0.235 & 0.046 & 0.002 & 0.001 & 0.030 & 0.010 \\
\hline $4 \mathrm{H}-6,125$ & 32.35 & 37.57 & 5.83 & 0.61 & 0.39 & 0.234 & 0.027 & 0.001 & 0.001 & 0.017 & 0.006 \\
\hline $5 \mathrm{H}-\mathrm{I}, 125$ & 34.35 & 40.15 & 6.26 & 0.61 & 0.43 & 0.258 & 0.063 & 0.001 & 0.005 & 0.039 & 0.014 \\
\hline $5 \mathrm{H}-2,125$ & 35.85 & 42.06 & 6.58 & 0.61 & 0.42 & 0.254 & 0.058 & 0.003 & 0.001 & 0.035 & 0.018 \\
\hline $5 \mathrm{H}-3,125$ & 37.35 & 44.09 & 6.91 & 0.61 & 0.32 & 0.191 & 0.009 & 0.003 & 0.000 & 0.006 & 0.002 \\
\hline $5 \mathrm{H}-4,125$ & 38.85 & 45.82 & 7.20 & 0.61 & 0.40 & 0.240 & 0.038 & 0.002 & 0.001 & 0.023 & 0.011 \\
\hline $5 \mathrm{H}-5,125$ & 40.35 & 47.49 & 7.42 & 0.79 & 0.46 & 0.362 & 0.113 & 0.001 & 0.005 & 0.071 & 0.031 \\
\hline $5 \mathrm{H}-6,125$ & 41.85 & 49.48 & 7.67 & 0.79 & 0.42 & 0.332 & 0.112 & 0.001 & 0.003 & 0.080 & 0.025 \\
\hline $6 \mathrm{H}-1,125$ & 43.85 & 51.52 & 8.26 & 0.07 & 0.45 & 0.034 & 0.015 & 0.000 & 0.000 & 0.011 & 0.004 \\
\hline $6 \mathrm{H}-2,125$ & 45.35 & 53.30 & 10.67 & 0.07 & 0.43 & 0.032 & 0.016 & 0.000 & 0.000 & 0.011 & 0.005 \\
\hline $6 \mathrm{H}-3,125$ & 46.85 & 54.58 & 12.41 & 0.07 & 0.61 & 0.045 & 0.022 & 0.000 & 0.000 & 0.014 & 0.007 \\
\hline $6 \mathrm{H}-4,125$ & 48.35 & 56.68 & 15.26 & 0.07 & 0.65 & 0.048 & 0.023 & 0.000 & 0.000 & 0.013 & 0.009 \\
\hline $6 \mathrm{H}-5,125$ & 49.85 & 64.22 & 25.47 & 0.07 & 0.47 & 0.035 & 0.014 & 0.000 & 0.000 & 0.007 & 0.007 \\
\hline $6 \mathrm{H}-6,125$ & 51.35 & 69.96 & 33.25 & 0.07 & 0.46 & 0.034 & 0.012 & 0.003 & 0.000 & 0.002 & 0.006 \\
\hline 145-886B- & & & & & & & & & & & \\
\hline $1 \mathrm{H}-1,50$ & 0.50 & 0.77 & 0.12 & 0.63 & 0.51 & 0.320 & 0.184 & 0.005 & 0.004 & 0.111 & 0.062 \\
\hline $1 \mathrm{H}-2,11$ & 1.31 & 2.76 & 0.44 & 0.63 & 0.47 & 0.294 & 0.179 & 0.002 & 0.005 & 0.105 & 0.063 \\
\hline $2 \mathrm{H}-1,50$ & 2.30 & 4.71 & 0.75 & 0.63 & 0.53 & 0.331 & 0.223 & 0.005 & 0.004 & 0.128 & 0.081 \\
\hline $2 \mathrm{H}-2,50$ & 3.80 & 4.76 & 0.76 & 0.63 & 0.48 & 0.301 & 0.202 & 0.003 & 0.005 & 0.111 & 0.077 \\
\hline $2 \mathrm{H}-3,50$ & 5.30 & 4.82 & 0.77 & 0.63 & 0.54 & 0.337 & 0.222 & 0.005 & 0.006 & 0.123 & 0.082 \\
\hline $2 \mathrm{H}-4,50$ & 6.80 & 4.87 & 0.78 & 0.63 & 0.47 & 0.294 & 0.190 & 0.002 & 0.008 & 0.102 & 0.072 \\
\hline $2 \mathrm{H}-5,50$ & 8.30 & 4.93 & 0.79 & 0.41 & 0.51 & 0.209 & 0.137 & 0.006 & 0.016 & 0.069 & 0.046 \\
\hline $2 \mathrm{H}-6,50$ & 9.80 & 4.98 & 0.80 & 0.41 & 0.48 & 0.196 & 0.132 & 0.003 & 0.004 & 0.073 & 0.049 \\
\hline $2 \mathrm{H}-7,45$ & 11.25 & 7.49 & 1.33 & 0.46 & 0.46 & 0.214 & 0.137 & 0.001 & 0.002 & 0.084 & 0.045 \\
\hline $3 \mathrm{H}-1,50$ & 11.80 & 9.11 & 1.68 & 0.46 & 0.45 & 0.210 & 0.122 & 0.001 & 0.001 & 0.074 & 0.041 \\
\hline $3 \mathrm{H}-2,50$ & 13.30 & 10.60 & 1.99 & 0.66 & 0.46 & 0.301 & 0.159 & 0.005 & 0.006 & 0.091 & 0.055 \\
\hline $3 \mathrm{H}-3,50$ & 14.80 & 12.61 & 2.30 & 0.66 & 0.48 & 0.317 & 0.172 & 0.002 & 0.002 & 0.099 & 0.065 \\
\hline $3 \mathrm{H}-4,50$ & 16.30 & 14.65 & 2.61 & 0.96 & 0.53 & 0.512 & 0.294 & 0.002 & 0.005 & 0.171 & 0.106 \\
\hline $3 \mathrm{H}-5,50$ & 17.80 & 16.15 & 2.76 & 0.96 & 0.39 & 0.371 & 0.078 & 0.001 & 0.000 & 0.043 & 0.029 \\
\hline $3 \mathrm{H}-6,50$ & 19.30 & 17.71 & 2.93 & 0.96 & 0.39 & 0.375 & 0.120 & 0.001 & 0.001 & 0.068 & 0.044 \\
\hline $4 \mathrm{H}-1,50$ & 21.30 & 20.47 & 3.21 & 0.96 & 0.42 & 0.408 & 0.103 & 0.001 & 0.000 & 0.058 & 0.039 \\
\hline $4 \mathrm{H}-2,50$ & 22.80 & 22.55 & 3.43 & 0.96 & 0.43 & 0.415 & 0.139 & 0.006 & 0.009 & 0.079 & 0.046 \\
\hline $4 \mathrm{H}-3,50$ & 24.30 & 24.59 & 3.71 & 0.61 & 0.31 & 0.185 & 0.021 & 0.000 & 0.000 & 0.012 & 0.007 \\
\hline $4 \mathrm{H}-4,50$ & 25.80 & 26.17 & 4.00 & 0.61 & 0.33 & 0.200 & 0.021 & 0.000 & 0.000 & 0.012 & 0.008 \\
\hline $4 \mathrm{H}-5,50$ & 27.30 & 27.52 & 4.25 & 0.61 & 0.32 & 0.197 & 0.015 & & 0.000 & 0.009 & 0.005 \\
\hline $4 \mathrm{H}-6,50$ & 28.80 & 28.87 & 4.49 & 0.61 & 0.33 & 0.200 & 0.013 & 0.001 & 0.000 & 0.008 & 0.004 \\
\hline $5 \mathrm{H}-1,50$ & 30.80 & 30.66 & 4.82 & 0.61 & 0.29 & 0.175 & 0.008 & 0.000 & 0.000 & 0.004 & 0.002 \\
\hline $5 \mathrm{H}-2,50$ & 32.30 & 32.01 & 5.07 & 0.61 & 0.32 & 0.193 & 0.024 & 0.009 & 0.002 & 0.015 & 0.006 \\
\hline $5 \mathrm{H}-3,50$ & 33.80 & 33.50 & 5.34 & 0.61 & 0.41 & 0.247 & 0.048 & 0.013 & 0.000 & 0.027 & 0.018 \\
\hline $5 \mathrm{H}-4,50$ & 35.30 & 35.00 & 5.62 & 0.61 & 0.33 & 0.199 & 0.037 & 0.011 & 0.003 & 0.018 & 0.012 \\
\hline $5 \mathrm{H}-5,50$ & 36.80 & 36.50 & 5.87 & 0.61 & 0.36 & 0.215 & 0.029 & 0.014 & 0.000 & 0.015 & 0.010 \\
\hline $5 \mathrm{H}-6,50$ & 38.30 & 38.00 & 6.07 & 0.61 & 0.35 & 0.214 & 0.028 & 0.017 & 0.001 & 0.016 & 0.010 \\
\hline $5 \mathrm{H}-7,50$ & 39.80 & 39.56 & 6.25 & 0.61 & 0.49 & 0.297 & 0.112 & 0.007 & 0.001 & 0.062 & 0.044 \\
\hline $6 \mathrm{H}-1,50$ & 40.30 & 40.39 & 6.35 & 0.61 & 0.42 & 0.254 & 0.059 & 0.014 & 0.001 & 0.032 & 0.023 \\
\hline $6 \mathrm{H}-2,50$ & 41.80 & 41.95 & 6.59 & 0.61 & 0.40 & 0.245 & 0.050 & 0.012 & 0.000 & 0.028 & 0.019 \\
\hline $6 \mathrm{H}-3,50$ & 43.30 & 43.45 & 6.82 & 0.61 & 0.36 & 0.220 & 0.024 & 0.022 & 0.000 & 0.014 & 0.009 \\
\hline $6 \mathrm{H}-4,50$ & 44.80 & 44.95 & 7.06 & 0.61 & 0.34 & 0.205 & 0.027 & 0.013 & 0.000 & 0.015 & 0.010 \\
\hline $6 \mathrm{H}-5,50$ & 46.30 & 46.45 & 7.29 & 0.79 & 0.40 & 0.317 & 0.063 & 0.007 & 0.001 & 0.031 & 0.025 \\
\hline $6 \mathrm{H}-6,52$ & 47.82 & 48.12 & 7.50 & 0.79 & 0.46 & 0.366 & 0.169 & 0.002 & 0.002 & 0.090 & 0.072 \\
\hline $6 \mathrm{H}-7,50$ & 49.30 & 50.28 & 7.77 & 0.79 & 0.44 & 0.350 & 0.139 & 0.003 & 0.001 & 0.073 & 0.061 \\
\hline $7 \mathrm{H}-1,47$ & 49.77 & 50.97 & 7.86 & 0.79 & 0.35 & 0.280 & 0.078 & 0.007 & 0.000 & 0.041 & 0.034 \\
\hline $7 \mathrm{H}-2,47$ & 51.27 & 53.02 & 10.29 & 0.07 & 0.44 & 0.033 & 0.019 & 0.000 & 0.000 & 0.010 & 0.008 \\
\hline $7 \mathrm{H}-3,47$ & 52.77 & 54.52 & 12.32 & 0.07 & 0.61 & 0.045 & 0.028 & 0.000 & 0.000 & 0.014 & 0.013 \\
\hline $7 \mathrm{H}-4,47$ & 54.27 & 55.55 & 13.72 & 0.07 & 0.65 & 0.048 & 0.019 & 0.000 & 0.001 & 0.010 & 0.007 \\
\hline $7 \mathrm{H}-5,47$ & 55.77 & 57.20 & 15.95 & 0.07 & 0.56 & 0.041 & 0.029 & 0.000 & 0.000 & 0.010 & 0.018 \\
\hline 7H-6, 47 & 57.27 & 58.79 & 18.11 & 0.07 & 0.52 & 0.039 & 0.030 & 0.000 & 0.000 & 0.010 & 0.016 \\
\hline $7 \mathrm{H}-7,47$ & 58.77 & 60.22 & 20.05 & 0.07 & 0.54 & 0.040 & 0.031 & 0.000 & 0.001 & 0.010 & 0.019 \\
\hline $8 X-1,50$ & 59.30 & 60.75 & 20.77 & 0.07 & 0.51 & 0.038 & 0.024 & 0.000 & 0.000 & 0.015 & 0.008 \\
\hline
\end{tabular}

Note: $\mathrm{LSR}=$ linear sedimentation rate, $\mathrm{MAR}=$ mass accumulation rate. $\mathrm{Blank}=$ no measurement. 


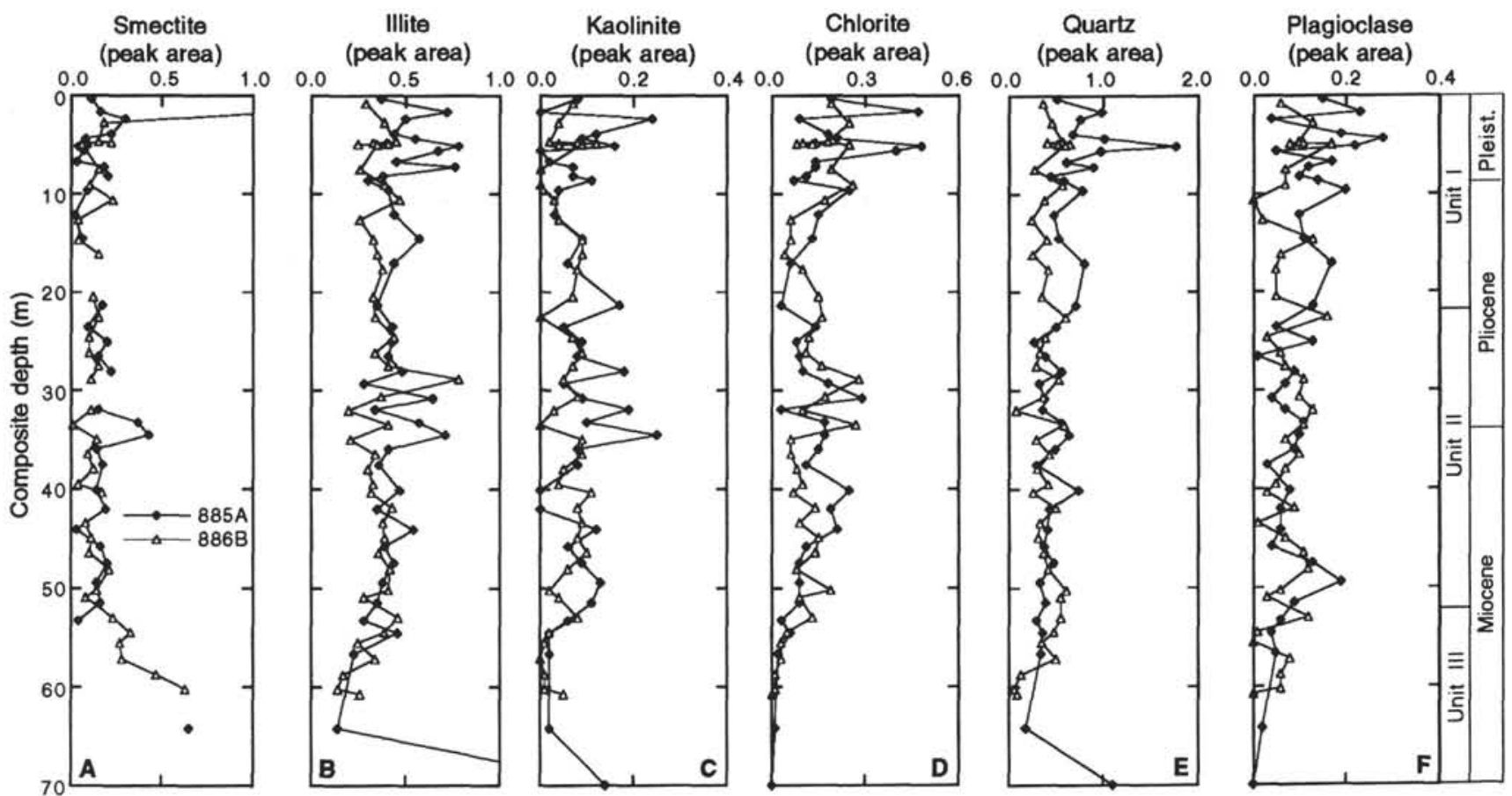

Figure 2. Mineral peak area for the $<2 \mu \mathrm{m}$ terrigenous size fraction vs. composite depth for Holes $885 \mathrm{~A}$ and $886 \mathrm{~B}$.
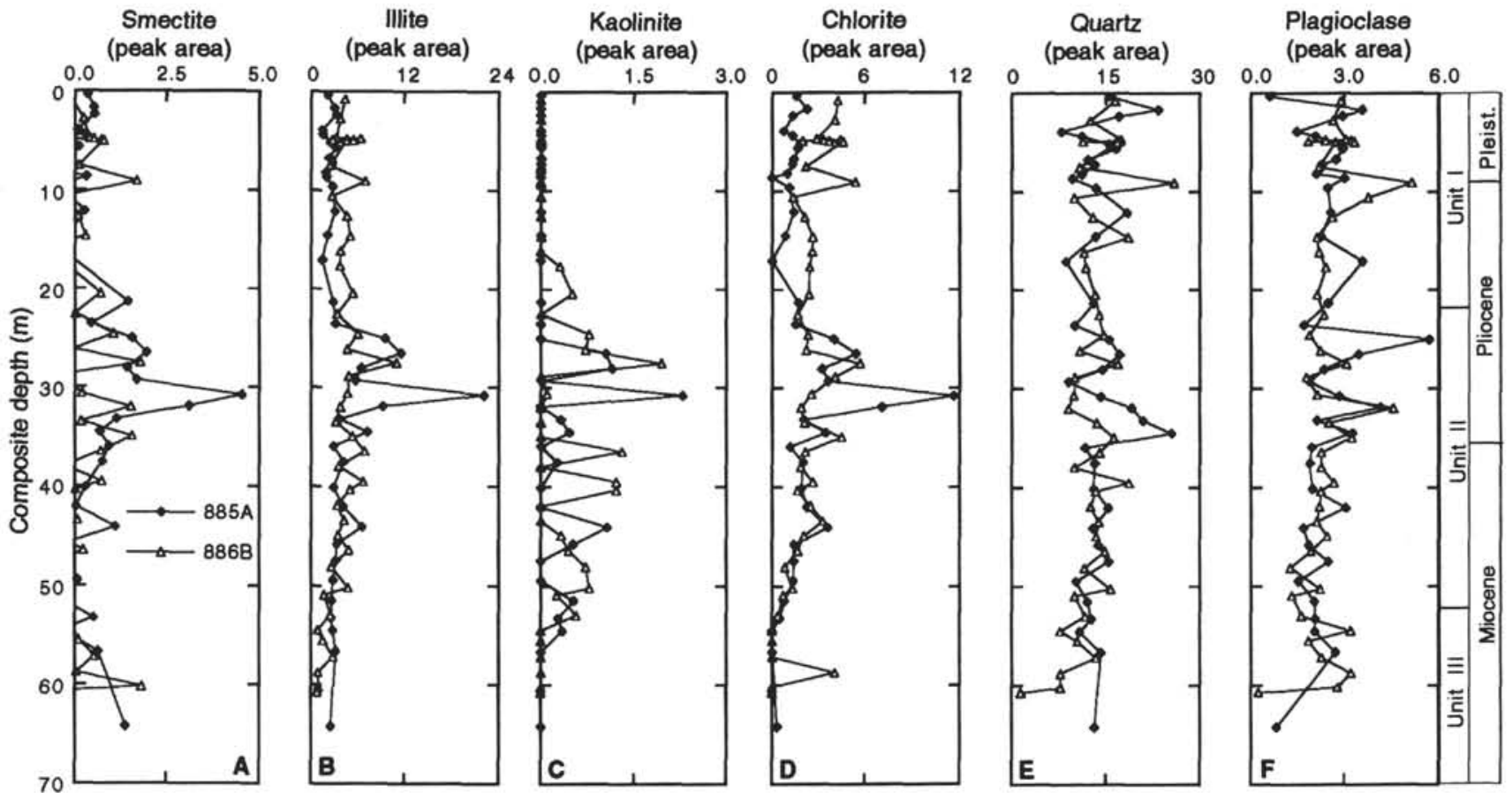

Figure 3. Mineral peak area for the $2-20 \mu \mathrm{m}$ terrigenous size fraction vs. composite depth for Holes $885 \mathrm{~A}$ and $886 \mathrm{~B}$.

decreases to uniformly low values in the diatom ooze. The concentration of hard magnetic minerals (such as goethite or hematite) does not explain the large magnetic concentrations indicated by the susceptibility or $X_{a r m}$ signal in Unit III. Thus, the magnetic carrier in these sediments must also include a softer component, such as magnetite. The concentration of hard-coercivity material begins to increase at the base of the uppermost red clay unit and displays a high-amplitude variability superimposed on a general HIRM increase throughout this unit. This increase in HIRM tracks the increase in susceptibility; thus, the magnetic carrier in this unit is harder than the sediments in the lower brown clay unit.

\section{Mineralogy}

Figures 2 and 3 illustrate the mineralogy for the $<2 \mu \mathrm{m}$ and $2-20$ $\mu \mathrm{m}$ terrigenous material at Holes Sites $885 \mathrm{~A}$ and $886 \mathrm{~B}$. Mineralogy was run at twice the sampling interval as the rock-magnetic measurements. A study to derive weighting factors for the $2-20 \mu \mathrm{m}$ size 
Table 3. Rock-magnetic measurements for Holes $885 \mathrm{~A}$ and 886B.

\begin{tabular}{|c|c|c|c|c|c|c|c|c|c|}
\hline $\begin{array}{l}\text { Core, section, } \\
\text { interval }(\mathrm{cm})\end{array}$ & $\begin{array}{l}\text { Depth } \\
\text { (mbsf) }\end{array}$ & $\begin{array}{l}\text { Composite } \\
\text { depth (m) }\end{array}$ & $\begin{array}{l}\text { Age } \\
\text { (Ma) }\end{array}$ & $\begin{array}{c}X_{A R M} \\
\left(10^{-6} \mathrm{~m}^{3} / \mathrm{g}\right)\end{array}$ & $\begin{array}{c}X_{t f} \\
\left(10^{-6} \mathrm{~m}^{3} / \mathrm{g}\right)\end{array}$ & $\begin{array}{c}X_{k f} \\
\left(10^{-6} \mathrm{~m}^{3 / g}\right)\end{array}$ & $X_{l y j} \sqrt{X_{l f}}$ & $X_{A R M^{\prime}} / X$ & $\begin{array}{c}\text { SIRM } \\
\left(10^{-6} \mathrm{~A} / \mathrm{m}^{2} / \mathrm{g}\right)\end{array}$ \\
\hline $145-885 \mathrm{~A}-$ & & & & & & & & & \\
\hline $\mathrm{IH}-1,25$ & 0.25 & 0.30 & 0.05 & 8.63 & 21.35 & 20.38 & 0.95 & 0.40 & 19089.01 \\
\hline $1 \mathrm{H}-1,50$ & 0.50 & 0.61 & 0.10 & 10.96 & 10.20 & 9.70 & 0.95 & 1.07 & 19310.96 \\
\hline $1 \mathrm{H}-1,125$ & 1.25 & 1.65 & 0.26 & 5.64 & 15.47 & 14.71 & 0.95 & 0.36 & 9846.23 \\
\hline IH-2, 25 & 1.75 & 2.34 & 0.37 & 11.27 & 17.67 & 16.70 & 0.95 & 0.64 & 17316.93 \\
\hline $1 \mathrm{H}-2,50$ & 2.00 & 2.68 & 0.43 & 11.92 & 15.15 & 14.41 & 0.95 & 0.79 & 24327.92 \\
\hline $1 \mathrm{H}-2,125$ & 2.75 & 3.89 & 0.62 & 14.90 & 27.68 & 26.30 & 0.95 & 0.54 & 30093.61 \\
\hline $1 \mathrm{H}-3,25$ & 3.25 & 4.34 & 0.69 & 10.04 & 10.57 & 9.98 & 0.94 & 0.95 & 10533.99 \\
\hline $1 \mathrm{H}-3,50$ & 3.50 & 4.49 & 0.72 & 13.62 & 7.22 & 6.79 & 0.94 & 1.89 & 13880.38 \\
\hline $1 \mathrm{H}-3,125$ & 4.25 & 5.16 & 0.84 & 9.92 & 16.05 & 15.22 & 0.95 & 0.62 & 14526.08 \\
\hline $2 \mathrm{H}-1,25$ & 4.85 & 5.63 & 0.93 & 12.89 & 25.96 & 24.18 & 0.93 & 0.50 & 23418.97 \\
\hline $2 \mathrm{H}-1,50$ & 5.10 & 5.88 & 0.98 & 14.93 & 17.06 & 15.90 & 0.93 & 0.88 & 29727.07 \\
\hline $2 \mathrm{H}-1,125$ & 5.85 & 6.72 & 1.16 & 9.91 & 13.45 & 12.64 & 0.94 & 0.74 & 11434.31 \\
\hline $2 \mathrm{H}-2,25$ & 6.35 & 7.24 & 1.27 & 9.29 & 14.78 & 14.09 & 0.95 & 0.63 & 11428.79 \\
\hline $2 \mathrm{H}-2,50$ & 6.60 & 7.49 & 1.33 & 14.92 & 8.57 & 8.07 & 0.94 & 1.74 & 15347.45 \\
\hline $2 \mathrm{H}-2,125$ & 7.35 & 8.19 & 1.48 & 15.71 & 29.69 & 28.27 & 0.95 & 0.53 & 29959.48 \\
\hline $2 \mathrm{H}-3,25$ & 7.85 & 8.63 & 1.57 & 14.70 & 37.85 & 35.59 & 0.94 & 0.39 & \\
\hline $2 \mathrm{H}-3,50$ & 8.10 & 8.85 & 1.62 & 15.09 & 16.37 & 15.55 & 0.95 & 0.92 & 29044.66 \\
\hline $2 \mathrm{H}-3,125$ & 8.85 & 9.61 & 1.78 & 11.14 & 13.64 & 12.78 & 0.94 & 0.82 & 13073.65 \\
\hline $2 \mathrm{H}-4,50$ & 9.60 & 10.62 & 2.00 & 16.27 & 13.86 & 13.17 & 0.95 & 1.17 & 30615.47 \\
\hline $2 \mathrm{H}-4,125$ & 10.35 & 12.12 & 2.23 & 10.94 & 12.08 & 11.36 & 0.94 & 0.91 & 9851.48 \\
\hline $2 \mathrm{H}-5,50$ & 11.10 & 13.71 & 2.47 & 18.89 & 7.26 & 6.88 & 0.95 & 2.60 & 12673.33 \\
\hline $2 \mathrm{H}-5,125$ & 11.85 & 14.55 & 2.60 & 14.00 & 8.33 & 7.72 & 0.93 & 1.68 & 7786.71 \\
\hline $2 \mathrm{H}-6,50$ & 12.60 & 15.75 & 2.72 & 13.66 & 2.99 & 2.82 & 0.94 & 4.57 & 6506.05 \\
\hline $2 \mathrm{H}-6,125$ & 13.35 & 17.06 & 2.86 & 15.14 & 16.91 & 16.02 & 0.95 & 0.90 & 19040.23 \\
\hline $2 \mathrm{H}-7.14$ & 13.74 & 17.49 & 2.90 & 23.77 & 19.94 & 18.75 & 0.94 & 1.19 & 44978.16 \\
\hline $3 \mathrm{H}-1,50$ & 14.60 & 19.54 & 3.12 & 12.09 & 1.90 & 1.73 & 0.91 & 6.36 & 4774.84 \\
\hline $3 \mathrm{H}-1,125$ & 15.35 & 21.35 & 3.30 & 16.14 & 6.44 & 5.90 & 0.92 & 2.51 & 6241.31 \\
\hline $3 \mathrm{H}-2,50$ & 16.10 & 22.80 & 3.45 & 12.03 & 3.67 & 3.40 & 0.93 & 3.28 & 4685.27 \\
\hline $3 \mathrm{H}-2,125$ & 16.85 & 23.55 & 3.53 & 11.74 & 2.62 & 2.31 & 0.88 & 4.48 & 3968.48 \\
\hline $3 \mathrm{H}-3,50$ & 17.60 & 24.30 & 3.65 & 9.06 & 1.15 & 1.06 & 0.92 & 7.88 & 3719.09 \\
\hline $3 \mathrm{H}-3,125$ & 18.35 & 25.05 & 3.79 & 5.20 & 1.20 & 1.12 & 0.94 & 4.33 & 2288.08 \\
\hline $3 \mathrm{H}-4,50$ & 19.10 & 25.80 & 3.93 & 8.81 & 1.39 & 1.27 & 0.92 & 6.34 & 4065.74 \\
\hline $3 \mathrm{H}-4,125$ & 19.85 & 26.55 & 4.07 & 8.19 & 1.36 & 1.17 & 0.86 & 6.02 & 2859.41 \\
\hline $3 \mathrm{H}-5,50$ & 20.60 & 27.30 & 4.21 & 9.12 & 0.89 & 0.80 & 0.90 & 10.25 & 3398.41 \\
\hline $3 \mathrm{H}-5,125$ & 21.35 & 28.05 & 4.34 & 7.30 & 0.90 & 0.80 & 0.89 & 8.11 & 2694.87 \\
\hline $3 \mathrm{H}-6.50$ & 22.10 & 28.74 & 4.47 & 6.12 & 0.36 & 0.31 & 0.87 & 17.00 & 2207.82 \\
\hline $3 \mathrm{H}-6,125$ & 22.85 & 29.31 & 4.57 & 5.29 & 0.31 & 0.31 & 1.00 & 17.06 & 2082.90 \\
\hline $3 \mathrm{H}-7,50$ & 23.60 & 29.87 & 4.68 & 0.60 & 0.40 & 0.37 & 0.93 & 1.50 & 1367.68 \\
\hline $4 \mathrm{H}-1,50$ & 24.10 & 30.25 & 4.75 & 0.11 & -0.13 & -0.12 & 0.93 & -0.85 & 156.25 \\
\hline $4 \mathrm{H}-1,125$ & 24.85 & 30.82 & 4.85 & 0.05 & -0.65 & -0.42 & 0.65 & -0.08 & 57.65 \\
\hline $4 \mathrm{H}-2,50$ & 25.60 & 31.38 & 4.96 & 0.12 & -0.08 & -0.14 & & -1.50 & 183.73 \\
\hline $4 \mathrm{H}-2,125$ & 26.35 & 31.95 & 5.06 & 4.01 & 1.31 & 1.22 & 0.93 & 3.06 & 4552.44 \\
\hline $4 \mathrm{H}-3,50$ & 27.10 & 32.58 & 5.18 & 0.11 & -0.09 & -0.09 & 1.00 & -1.22 & 244.51 \\
\hline $4 \mathrm{H}-3,125$ & 27.85 & 33.23 & 5.30 & 5.99 & 1.45 & 1.40 & 0.97 & 4.13 & 2496.23 \\
\hline $4 \mathrm{H}-4,50$ & 28.60 & 33.88 & 5.42 & 0.09 & 0.05 & 0.00 & & 1.80 & 197.96 \\
\hline $4 \mathrm{H}-4,125$ & 29.35 & 34.53 & 5.53 & 0.11 & 0.01 & -0.02 & & 11.00 & 241.65 \\
\hline $4 \mathrm{H}-5,50$ & 30.10 & 35.21 & 5.66 & 0.06 & 1.33 & 1.28 & 0.96 & 0.05 & 5004.97 \\
\hline $4 \mathrm{H}-5,125$ & 30.85 & 35.99 & 5.79 & 8.71 & 2.65 & 2.55 & 0.96 & 3.29 & 4341.91 \\
\hline $4 \mathrm{H}-6,50$ & 31.60 & 36.78 & 5.92 & 8.37 & 0.94 & 0.94 & 0.99 & 8.90 & 3932.85 \\
\hline $4 \mathrm{H}-6,125$ & 32.35 & 37.57 & 6.02 & 7.35 & 2.00 & 1.83 & 0.92 & 3.68 & 2875.38 \\
\hline $4 \mathrm{H}-7,50$ & 33.10 & 38.44 & 6.12 & 10.61 & 1.60 & 1.50 & 0.93 & 6.63 & 3955.20 \\
\hline $5 \mathrm{H}-1,50$ & 33.60 & 39.40 & 6.23 & 21.14 & 3.95 & 3.68 & 0.93 & 5.35 & 7242.45 \\
\hline $5 \mathrm{H}-\mathrm{I}, 125$ & 34.35 & 40.15 & 6.32 & 13.16 & 3.50 & 3.36 & 0.96 & 3.76 & 5710.37 \\
\hline $5 \mathrm{H}-2,50$ & 35.10 & 40.99 & 6.44 & 8.21 & 0.85 & 0.70 & 0.83 & 9.66 & 2759.93 \\
\hline $5 \mathrm{H}-2,125$ & 35.85 & 42.06 & 6.61 & 10.24 & 3.40 & 3.13 & 0.92 & 3.01 & 3959.29 \\
\hline $5 \mathrm{H}-3,50$ & 36.60 & 43.23 & 6.79 & 15.80 & 3.13 & 2.87 & 0.92 & 5.05 & 5803.21 \\
\hline $5 \mathrm{H}-3,125$ & 37.35 & 44.09 & 6.93 & 2.83 & 0.24 & 0.20 & 0.86 & 11.79 & 1026.12 \\
\hline $5 \mathrm{H}-4,50$ & 38.10 & 44.96 & 7.06 & 6.99 & 1.15 & 1.11 & 0.96 & 6.08 & 2935.40 \\
\hline $5 \mathrm{H}-4,125$ & 38.85 & 45.82 & 7.20 & 7.35 & 2.13 & 1.96 & 0.92 & 3.45 & 2756.58 \\
\hline $5 \mathrm{H}-5,50$ & 39.60 & 46.66 & 7.31 & 14.70 & 2.50 & 2.27 & 0.91 & 5.88 & 5134.13 \\
\hline $5 \mathrm{H}-5,125$ & 40.35 & 47.49 & 7.42 & 14.49 & 6.45 & 5.95 & 0.92 & 2.25 & 6258.31 \\
\hline $5 \mathrm{H}-6,50$ & 41.10 & 48.46 & 7.54 & 23.78 & 5.28 & 4.78 & 0.91 & 4.50 & 7885.38 \\
\hline $5 \mathrm{H}-6,125$ & 41.85 & 49.48 & 7.67 & 14.86 & 6.06 & 5.60 & 0.92 & 2.45 & 5847.84 \\
\hline $5 \mathrm{H}-7,50$ & 42.60 & 50.32 & 7.77 & 21.01 & 4.94 & 4.54 & 0.92 & 4.25 & 7309.48 \\
\hline $6 \mathrm{H}-1,50$ & 43.10 & 50.84 & 7.84 & 14.93 & 3.36 & 3.07 & 0.91 & 4.44 & 5903.40 \\
\hline $6 \mathrm{H}-1,125$ & 43.85 & 51.52 & 8.26 & 15.01 & 8.67 & 7.96 & 0.92 & 1.73 & 6564.84 \\
\hline $6 \mathrm{H}-2,50$ & 44.60 & 52.61 & 9.74 & 27.25 & 16.74 & 15.67 & 0.94 & 1.63 & 36453.07 \\
\hline $6 \mathrm{H}-2,125$ & 45.35 & 53.30 & 10.67 & 27.53 & 11.94 & 10.81 & 0.91 & 2.31 & 8779.16 \\
\hline $6 \mathrm{H}-3,50$ & 46,10 & 53.94 & 11.54 & 26.98 & 7.69 & 7.05 & 0.92 & 3.51 & 11360.63 \\
\hline $6 \mathrm{H}-3,125$ & 46.85 & 54.58 & 12.41 & 27.13 & 17.88 & 16.24 & 0.91 & 1.52 & 8482.88 \\
\hline $6 \mathrm{H}-4,50$ & 47.60 & 55.22 & 13.28 & 47.08 & 17.42 & 15.70 & 0.90 & 2.70 & 14663.52 \\
\hline $6 \mathrm{H}-4,125$ & 48.35 & 56.68 & 15.26 & 37.98 & 28.36 & 25.65 & 0.90 & 1.34 & 11938.51 \\
\hline $6 \mathrm{H}-5,50$ & 49.10 & 59.68 & 19.31 & 84.97 & 38.89 & 35.87 & 0.92 & 2.18 & 40982.34 \\
\hline $6 \mathrm{H}-5,125$ & 49.85 & 64.22 & 25.47 & 54.57 & 41.99 & 38.80 & 0.92 & 1.30 & 23838.75 \\
\hline $6 \mathrm{H}-6,50$ & 50.60 & 69.03 & 31.99 & 52.74 & 27.11 & 24.88 & 0.92 & 1.95 & 19382.38 \\
\hline $6 \mathrm{H}-6,125$ & 51.35 & 69.96 & 33.25 & 18.32 & 26.06 & 24.39 & 0.94 & 0.70 & 8056.40 \\
\hline $6 \mathrm{H}-7,37$ & 51.85 & 70.45 & 33.91 & 23.27 & 17.14 & 15.82 & 0.92 & 1.36 & 9258.05 \\
\hline $145-886 \mathrm{~B}-$ & & & & & & & & & \\
\hline $1 \mathrm{H}-1,50$ & 0.50 & 0.77 & 0.12 & 9.35 & 10.37 & 9.93 & 0.96 & 0.90 & 17185.43 \\
\hline $1 \mathrm{H}-2,11$ & 1.31 & 2.76 & 0.44 & 10.16 & 11.26 & 10.72 & 0.95 & 0.90 & 17444.17 \\
\hline $2 \mathrm{H}-1,50$ & 2.30 & 4.71 & 0.75 & 14.10 & 10.73 & 10.14 & 0.95 & 1.31 & 17927.93 \\
\hline $2 \mathrm{H}-1,125$ & 3.05 & 4.73 & 0.76 & 12.69 & 23.31 & 22.03 & 0.95 & 0.54 & 18983.51 \\
\hline $2 \mathrm{H}-2,50$ & 3.80 & 4.76 & 0.76 & 10.61 & 9.94 & 9.46 & 0.95 & 1.07 & 14056.10 \\
\hline $2 \mathrm{H}-2,125$ & 4.55 & 4.79 & 0.77 & 9.43 & 12.77 & 12.23 & 0.96 & 0.74 & 9530.20 \\
\hline $2 \mathrm{H}-3,50$ & 5.30 & 4.82 & 0.77 & 12.41 & 9.12 & 8.63 & 0.95 & 1.36 & 14578.18 \\
\hline $2 \mathrm{H}-3,125$ & 6.05 & 4.85 & 0.77 & 11.13 & 17.29 & 16.65 & 0.96 & 0.64 & 12194.59 \\
\hline $2 \mathrm{H}-4,50$ & 6.80 & 4.87 & 0.78 & 16.98 & 22.33 & 21.32 & 0.95 & 0.76 & \\
\hline
\end{tabular}


Table 3 (continued).

\begin{tabular}{|c|c|c|c|c|c|c|c|c|c|c|c|c|}
\hline $\begin{array}{l}\text { Core, section, } \\
\text { interval }(\mathrm{cm})\end{array}$ & $\begin{array}{l}\text { Depth } \\
\text { (mbsf) }\end{array}$ & $\begin{array}{l}\text { Composite } \\
\text { depth }(m)\end{array}$ & $\begin{array}{l}\text { Age } \\
(\mathrm{Ma})\end{array}$ & $\begin{array}{c}X_{A R M} \\
\left(10^{-6} \mathrm{~m}^{3} / \mathrm{g}\right)\end{array}$ & $\begin{array}{c}X_{l f} \\
\left(10^{-6} \mathrm{~m}^{3} / \mathrm{g}\right)\end{array}$ & $\begin{array}{c}X_{h f} \\
\left(10^{-6} \mathrm{~m}^{3} / \mathrm{g}\right)\end{array}$ & $X_{h f} / X_{l f}$ & $X_{A R M^{\prime}} / X$ & $\begin{array}{c}\text { SIRM } \\
\left(10^{-6} \mathrm{~A} / \mathrm{m}^{2} / \mathrm{g}\right)\end{array}$ & $\begin{array}{c}I R M_{-0.3 T} \\
\left(10^{-6} \mathrm{~A} / \mathrm{m}^{2} / \mathrm{g}\right)\end{array}$ & S-ratio & $\begin{array}{c}\text { HIRM } \\
\left(10^{-6} \mathrm{~A} / \mathrm{m}^{2} / \mathrm{g}\right)\end{array}$ \\
\hline $2 \mathrm{H}-4,125$ & 7.55 & 4.90 & 0.78 & 14.26 & 34.00 & 32.66 & 0.96 & 0.42 & 35309.86 & -32072.44 & 0.91 & 957.76 \\
\hline $2 \mathrm{H}-5,50$ & 8.30 & 4.93 & 0.79 & 12.44 & 12.85 & 12.33 & 0.96 & 0.97 & 18910.29 & -17644.32 & 0.93 & 366.28 \\
\hline $2 \mathrm{H}-5,125$ & 9.05 & 4.96 & 0.80 & 11.12 & 31.28 & 30.39 & 0.97 & 0.36 & 21191.52 & -19599.23 & 0.92 & 602.74 \\
\hline $2 \mathrm{H}-6,50$ & 9.80 & 4.98 & 0.80 & 12.56 & 8.79 & 8.29 & 0.94 & 1.43 & 16163.62 & -14944.65 & 0.92 & 338.88 \\
\hline $2 \mathrm{H}-6,125$ & 10.55 & 5.28 & 0.86 & 9.15 & 14.31 & 13.71 & 0.96 & 0.64 & 13160.71 & -11398.96 & 0.87 & 551.41 \\
\hline $2 \mathrm{H}-7,45$ & 11.25 & 7.49 & 1.33 & 12.75 & 9.24 & 8.63 & 0.93 & 1.38 & 16088.10 & -14904.64 & 0.93 & 323.02 \\
\hline $3 \mathrm{H}-1,50$ & 11.80 & 9.11 & 1.68 & 17.26 & 15.91 & 14.97 & 0.94 & 1.08 & 29031.97 & -26521.77 & 0.91 & 690.77 \\
\hline $3 \mathrm{H}-1,125$ & 12.55 & 9.83 & 1.83 & 11.33 & 13.99 & 13.32 & 0.95 & 0.81 & 16430.93 & -14653.08 & 0.89 & 425.22 \\
\hline $3 \mathrm{H}-2,50$ & 13.30 & 10.60 & 1.99 & 15.60 & 8.73 & 8.16 & 0.93 & 1.79 & 13796.14 & -12691.30 & 0.92 & 320.15 \\
\hline $3 \mathrm{H}-2,125$ & 14.05 & 11.56 & 2.14 & 11.32 & 9.56 & 8.99 & 0.94 & 1.18 & 8953.88 & -8229.79 & 0.92 & 198.31 \\
\hline $3 \mathrm{H}-3,50$ & 14.80 & 12.61 & 2.30 & 17.43 & 9.43 & 8.87 & 0.94 & 1.85 & 14687.23 & -13732.89 & 0.94 & 304.93 \\
\hline $3 \mathrm{H}-3,125$ & 15.55 & 13.67 & 2.46 & 15.80 & 10.86 & 10.34 & 0.95 & 1.45 & 10376.44 & -9819.99 & 0.95 & 155.64 \\
\hline $3 \mathrm{H}-4,50$ & 16.30 & 14.65 & 2.61 & 12.82 & 2.85 & 2.68 & 0.94 & 4.50 & 6286.19 & -5977.47 & 0.95 & 71.35 \\
\hline $3 \mathrm{H}-4,125$ & 17.05 & 15.40 & 2.69 & 14.45 & 8.65 & 8.14 & 0.94 & 1.67 & 7834.02 & -7264.36 & 0.93 & 160.45 \\
\hline $3 \mathrm{H}-5,50$ & 17.80 & 16.15 & 2.76 & 17.35 & 4.59 & 4.25 & 0.93 & 3.78 & 10103.46 & -9426.28 & 0.93 & 158.27 \\
\hline $3 \mathrm{H}-5,125$ & 18.55 & 16.90 & 2.84 & 13.74 & 13.93 & 13.33 & 0.96 & 0.99 & 18838.13 & -17645.96 & 0.94 & 277.75 \\
\hline $3 \mathrm{H}-6,50$ & 19.30 & 17.71 & 2.93 & 15.83 & 3.19 & 2.94 & 0.92 & 4.96 & 6085.17 & -5831.82 & 0.96 & 64.42 \\
\hline $3 \mathrm{H}-6,125$ & 20.05 & 18.75 & 3.03 & 11.64 & 5.09 & 4.81 & 0.94 & 2.29 & 5585.70 & -5048.87 & 0.90 & 129.74 \\
\hline $4 \mathrm{H}-1,50$ & 21.30 & 20.47 & 3.21 & 16.96 & 5.62 & 5.32 & 0.95 & 3.02 & 10604.85 & -10294.70 & 0.97 & 80.19 \\
\hline $4 \mathrm{H}-1,125$ & 22.05 & 21.51 & 3.32 & 11.68 & 2.80 & 2.58 & 0.92 & 4.17 & 4271.97 & -4224.29 & 0.99 & 9.79 \\
\hline $4 \mathrm{H}-2,50$ & 22.80 & 22.55 & 3.43 & 8.68 & 0.89 & 0.80 & 0.89 & 9.75 & 3189.68 & -3120.39 & 0.98 & 12.69 \\
\hline $4 \mathrm{H}-2,125$ & 23.55 & 23,66 & 3.54 & 10.28 & 2.36 & 2.18 & 0.92 & 4.36 & 4384.55 & -4196.38 & 0.96 & 38.31 \\
\hline $4 \mathrm{H}-3,50$ & 24.30 & 24.59 & 3.71 & 11.15 & 1.18 & 1.15 & 0.98 & 9.45 & 4056.34 & -3926.32 & 0.97 & 25.75 \\
\hline $4 \mathrm{H}-3,125$ & 25.05 & 25.50 & 3.87 & 7.70 & 3.28 & 3.13 & 0.95 & 2.35 & 7027.44 & -6476.59 & 0.92 & 97.95 \\
\hline $4 \mathrm{H}-4,50$ & 25.80 & 26.17 & 4.00 & 7.11 & 1.24 & 1.10 & 0.89 & 5.73 & 4612.98 & -4508.74 & 0.98 & 20.32 \\
\hline $4 \mathrm{H}-4,125$ & 26.55 & 26.85 & 4.12 & 6.96 & 0.68 & 0.59 & 0.87 & 10.24 & 2623.39 & -2567.60 & 0.98 & 9.66 \\
\hline $4 \mathrm{H}-5,50$ & 27.30 & 27.52 & 4.25 & 0.20 & -0.12 & -0.12 & 1.01 & -1.67 & 256.05 & -224.98 & 0.88 & 6.16 \\
\hline $4 \mathrm{H}-5,125$ & 28.05 & 28.19 & 4.37 & 4.04 & 0.08 & 0.09 & 1.15 & 50.50 & 1498.50 & -1431.61 & 0.96 & 11.33 \\
\hline $4 \mathrm{H}-6,50$ & 28.80 & 28.87 & 4.49 & 0.07 & -0.22 & -0.27 & 1.23 & -0.32 & 91.12 & -72.59 & 0.80 & 3.22 \\
\hline $5 \mathrm{H}-1,50$ & 30.80 & 30.66 & 4.82 & 6.58 & 2.69 & 2.57 & 0.95 & 2.45 & 11340.34 & -10902.72 & 0.96 & 83.55 \\
\hline $5 \mathrm{H}-1,125$ & 31.55 & 31.34 & 4.95 & 0.08 & -0.29 & -0.31 & 1.05 & -0.28 & 129.30 & -94.73 & 0.73 & 5.25 \\
\hline $5 \mathrm{H}-2,50$ & 32.30 & 32.01 & 5.07 & 0.15 & 0.33 & 0.28 & 0.83 & 0.45 & 487.65 & -401.00 & 0.82 & 21.22 \\
\hline $5 \mathrm{H}-2,125$ & 33.05 & 32.75 & 5.21 & 0.08 & 0.00 & -0.03 & 0.00 & & 176.52 & -125.29 & 0.71 & 9.77 \\
\hline $5 \mathrm{H}-3,50$ & 33.80 & 33.50 & 5.34 & 8.32 & 3.98 & 3.82 & 0.96 & 2.09 & 13243.68 & -12814.12 & 0.97 & 84.70 \\
\hline $5 \mathrm{H}-3,125$ & 34.55 & 34.25 & 5.48 & 0.09 & 0.03 & 0.07 & 2.01 & 3.00 & 265.94 & -202.86 & 0.76 & 15.89 \\
\hline $5 \mathrm{H}-4,50$ & 35.30 & 35.00 & 5.62 & 6.65 & 0.84 & 0.79 & 0.94 & 7.92 & 3477.03 & -3304.73 & 0.95 & 36.75 \\
\hline $5 \mathrm{H}-4,125$ & 36.05 & 35.75 & 5.75 & 5.83 & 0.95 & 0.94 & 0.99 & 6.14 & 3552.57 & -3381.03 & 0.95 & 33.45 \\
\hline $5 \mathrm{H}-5,50$ & 36.80 & 36.50 & 5.87 & 9.61 & 2.86 & 2.67 & 0.93 & 3.36 & 6917.48 & -6598.62 & 0.95 & 67.57 \\
\hline $5 \mathrm{H}-5,125$ & 37.55 & 37.25 & 5.98 & 6.92 & 2.43 & 2.24 & 0.92 & 2.85 & 3034.91 & -2854.74 & 0.94 & 45.93 \\
\hline $5 \mathrm{H}-6,50$ & 38.30 & 38.00 & 6.07 & 10.54 & 3.05 & 2.95 & 0.97 & 3.46 & 8000.02 & -7544.79 & 0.94 & 134.10 \\
\hline $5 \mathrm{H}-6,125$ & 39.05 & 38.75 & 6.16 & 5.44 & 1.56 & 1.53 & 0.98 & 3.49 & 3406.59 & -3234.32 & 0.95 & 48.48 \\
\hline $5 \mathrm{H}-7,50$ & 39.80 & 39.56 & 6.25 & 14.63 & 2.64 & 2.39 & 0.91 & 5.54 & 5134.38 & -4920.78 & 0.96 & 53.69 \\
\hline $6 \mathrm{H}-1.50$ & 40.30 & 40.39 & 6.35 & 10.78 & 1.88 & 1.72 & 0.91 & 5.73 & 4030.02 & -3825.57 & 0.95 & 49.61 \\
\hline $6 \mathrm{H}-1,125$ & 41.05 & 41.20 & 6.47 & 6.94 & 1.32 & 1.20 & 0.91 & 5.26 & 2482.49 & -2368.66 & 0.95 & 23.76 \\
\hline $6 \mathrm{H}-2,50$ & 41.80 & 41.95 & 6.59 & 3.30 & 0.43 & 0.39 & 0.90 & 7.67 & 1429.96 & -1406.73 & 0.98 & 5.07 \\
\hline $6 \mathrm{H}-2,125$ & 42.55 & 42.70 & 6.71 & 8.10 & 2.00 & 1.87 & 0.93 & 4.05 & 2946.94 & -2813.81 & 0.95 & 30.23 \\
\hline $6 \mathrm{H}-3,50$ & 43.30 & 43.45 & 6.82 & 5.47 & 0.58 & 0.53 & 0.91 & 9.43 & 2355.29 & -2242.49 & 0.95 & 22.90 \\
\hline $6 \mathrm{H}-3,125$ & 44.05 & 44.20 & 6.94 & 3.27 & 0.15 & 0.10 & 0.70 & 21.80 & 1121.57 & -1147.82 & 1.02 & -3.94 \\
\hline $6 \mathrm{H}-4,50$ & 44.80 & 44.95 & 7.06 & 11.00 & 1.85 & 1.72 & 0.93 & 5.95 & 4050.49 & -3859.19 & 0.95 & 45.85 \\
\hline $6 \mathrm{H}-4,125$ & 45.55 & 45.70 & 7.18 & 6.95 & 1.71 & 1.56 & 0.92 & 4.06 & 2528.16 & -2382.24 & 0.94 & 34.45 \\
\hline $6 \mathrm{H}-5,50$ & 46.30 & 46.45 & 7.29 & 22.24 & 5.24 & 4.84 & 0.92 & 4.24 & 7610.02 & -7285.45 & 0.96 & 89.77 \\
\hline $6 \mathrm{H}-5,125$ & 47.05 & 47.20 & 7.38 & 14.85 & 6.34 & 5.87 & 0.93 & 2.34 & 5929.67 & -5548.89 & 0.94 & 109.11 \\
\hline $6 \mathrm{H}-6,52$ & 47.82 & 48.12 & 7.50 & 15.92 & 4.06 & 3.72 & 0.92 & 3.92 & 6217.09 & -5826.15 & 0.94 & 103.28 \\
\hline $6 \mathrm{H}-6,125$ & 48.55 & 49.19 & 7.63 & 15.74 & 8.48 & 8.01 & 0.94 & 1.86 & 8553.62 & -8049.89 & 0.94 & 138.80 \\
\hline $6 \mathrm{H}-7.50$ & 49.30 & 50.28 & 7.77 & 12.70 & 2.46 & 2.29 & 0.93 & 5.16 & 4781.84 & -4597.94 & 0.96 & 38.92 \\
\hline $7 \mathrm{H}-1,47$ & 49.77 & 50.97 & 7.86 & 29.39 & 8.58 & 8.05 & 0.94 & 3.43 & 10269.02 & -9895.73 & 0.96 & 99.26 \\
\hline $7 \mathrm{H}-1,125$ & 50.55 & 52.11 & 9.06 & 20.91 & 9.36 & 8.60 & 0.92 & 2.23 & 7612.39 & -7277.04 & 0.96 & 88.16 \\
\hline $7 \mathrm{H}-2,47$ & 51.27 & 53.02 & 10.29 & 31.59 & 11.87 & 10.83 & 0.91 & 2.66 & 10230.93 & -9924.38 & 0.97 & 111.66 \\
\hline $7 \mathrm{H}-2,125$ & 52.05 & 53.80 & 11.35 & 28.92 & 13.24 & 12.21 & 0.92 & 2.18 & 9553.70 & -9177.02 & 0.96 & 100.12 \\
\hline $7 \mathrm{H}-3,47$ & 52.77 & 54.52 & 12.32 & 47.93 & 21.45 & 19.57 & 0.91 & 2.23 & 16079.16 & -15695.98 & 0.98 & 150.50 \\
\hline $7 \mathrm{H}-3,125$ & 53.55 & 55.30 & 13.38 & 36.20 & 24.57 & 22.56 & 0.92 & 1.47 & 12257.41 & -11916.47 & 0.97 & 123.74 \\
\hline $7 \mathrm{H}-4,47$ & 54.27 & 55.55 & 13.72 & 62.44 & 20.90 & 18.95 & 0.91 & 2.99 & 19881.76 & -19313.51 & 0.97 & 189.67 \\
\hline $7 \mathrm{H}-4,125$ & 55.05 & 56.33 & 14.77 & 48.78 & 35.41 & 32.42 & 0.92 & 1.38 & 17306.08 & -16839.75 & 0.97 & 168.86 \\
\hline $7 \mathrm{H}-5,47$ & 55.77 & 57.20 & 15.95 & 71.37 & 36.79 & 33.98 & 0.92 & 1.94 & 30391.77 & -29341.97 & 0.97 & 329.42 \\
\hline $7 \mathrm{H}-5,125$ & 56.55 & 58.14 & 17.23 & 55.03 & 50.35 & 46.72 & 0.93 & 1.09 & 23183.52 & -22028.97 & 0.95 & 400.63 \\
\hline $7 \mathrm{H}-6,47$ & 57.27 & 58.79 & 18.11 & 105.39 & 62.71 & 58.37 & 0.93 & 1.68 & & & & \\
\hline $7 \mathrm{H}-6,130$ & 58.10 & 59.55 & 19.14 & 65.62 & 84.80 & 79.70 & 0.94 & 0.77 & & & & \\
\hline $7 \mathrm{H}-7,47$ & 58.77 & 60.22 & 20.05 & 38.72 & 35.86 & 32.75 & 0.91 & 1.08 & 15423.40 & -15155.70 & 0.98 & 82.57 \\
\hline $8 X-1,50$ & 59.30 & 60.75 & 20.77 & 11.03 & 9.74 & 9.22 & 0.95 & 1.13 & 17720.03 & -16517.44 & 0.93 & 339.62 \\
\hline
\end{tabular}

Notes: $X_{A R M}=$ anhysteretic remanent magnetism, $X_{V f}=$ low-frequency susceptibility, $X_{h f}=$ high-frequency susceptibility, $X_{h f} / X_{I f}=$ frequency ratio, $X_{A R M} / X=$ grain-size ratio, $S I R M=$ saturated isothermal remanent magnetization, $I R M_{-0,3 T}=$ isothermal remanent magnetization. Blank = no measurement.

fraction has not yet been completed, so normalized peak areas are presented instead of absolute weight percent (Tables 4, 5). The absolute values of the peak areas are only comparable for the individual mineral within each size fraction. Relative change is directly comparable among all minerals and size classes.

\section{Smectite}

Smectite is produced by continental weathering processes, weathering of volcanogenic material, and authigenic formation in the sediment column; so the interpretation of this mineral group is complex. However, Chamley (1989) states that most of the smectite in pelagic deep-sea sediments may be interpreted as continental weathering products. The concentration of smectite is relatively uniform in the $<2$ $\mu \mathrm{m}$ size fraction in both cores and through all units (Fig. 2A). For the 2-20 $\mu \mathrm{m}$ size fraction (Fig. 3A), smectite shows about a threefold increase in peak area between approximately 25 and $35 \mathrm{mcd}$ and is relatively uniform and present in equal amounts in both stratigraphic clay Units I and III.

\section{Illite}

Illite is a ubiquitous terrigenous weathering product, generally associated with cool, dry environments, that is unlikely to form authi- 
Table 4. Normalized mineral peak areas for the $<2 \mu \mathrm{m}$ fraction of Holes $885 \mathrm{~A}$ and $886 \mathrm{~B}$.

\begin{tabular}{|c|c|c|c|c|c|c|c|c|c|}
\hline $\begin{array}{l}\text { Core, section, } \\
\text { interval }(\mathrm{cm})\end{array}$ & $\begin{array}{l}\text { Depth } \\
\text { (mbsf) }\end{array}$ & $\begin{array}{l}\text { Composite } \\
\text { depth }(m)\end{array}$ & $\begin{array}{l}\text { Age } \\
\text { (Ma) }\end{array}$ & $\begin{array}{l}\text { Smectite } \\
\text { peak area }\end{array}$ & $\begin{array}{c}\text { Illite } \\
\text { peak area }\end{array}$ & $\begin{array}{l}\text { Kaolinite } \\
\text { peak area }\end{array}$ & $\begin{array}{c}\text { Chlorite } \\
\text { peak area }\end{array}$ & $\begin{array}{c}\text { Quartz } \\
\text { peak area }\end{array}$ & $\begin{array}{c}\text { Plagioclase } \\
\text { peak area }\end{array}$ \\
\hline \multicolumn{10}{|l|}{$145-885 \mathrm{~A}-$} \\
\hline $1 \mathrm{H}-1,25$ & 0.25 & 0.30 & 0.05 & 0.11 & 0.37 & 0.08 & 0.19 & 0.51 & 0.15 \\
\hline $1 \mathrm{H}-1,125$ & 1.25 & 1.65 & 0.26 & 0.16 & 0.72 & 0.00 & 0.47 & 0.98 & 0.23 \\
\hline $1 \mathrm{H}-2,25$ & 1.75 & 2.34 & 0.37 & 0.30 & 0.50 & 0.24 & 0.09 & 0.76 & 0.04 \\
\hline IH-2, 125 & 2.75 & 3.89 & 0.62 & 0.22 & 0.44 & 0.12 & 0.18 & 0.68 & 0.19 \\
\hline IH-3, 25 & 3.25 & 4.34 & 0.69 & 0.08 & 0.55 & 0.09 & 0.21 & 1.01 & 0.28 \\
\hline IH $-3,125$ & 4.25 & 5.16 & 0.85 & 0.04 & 0.78 & 0.16 & 0.48 & 1.76 & 0.22 \\
\hline $2 \mathrm{H}-1,25$ & 4.85 & 5.63 & 0.96 & 0.07 & 0.67 & 0.00 & 0.40 & 0.97 & 0.05 \\
\hline $2 \mathrm{H}-1,125$ & 5.85 & 6.72 & 1.11 & 0.03 & 0.45 & 0.02 & 0.14 & 0.61 & 0.17 \\
\hline $2 \mathrm{H}-2,25$ & 6.35 & 7.24 & 1.27 & 0.18 & 0.76 & 0.07 & 0.14 & 0.89 & 0.12 \\
\hline $2 \mathrm{H}-2,125$ & 7.35 & 8.19 & 1.48 & 0.20 & 0.38 & 0.07 & 0.11 & 0.44 & 0.10 \\
\hline $2 \mathrm{H}-3,25$ & 7.85 & 8.63 & 1.57 & 0.00 & 0.30 & 0.11 & 0.07 & 0.58 & 0.14 \\
\hline $2 \mathrm{H}-3,125$ & 8.85 & 9.61 & 1.78 & 0.09 & 0.41 & 0.04 & 0.25 & 0.78 & 0.20 \\
\hline $2 \mathrm{H}-4,125$ & 10.35 & 12.12 & 2.23 & 0.02 & 0.44 & 0.03 & 0.15 & 0.48 & 0.10 \\
\hline $2 \mathrm{H}-5,125$ & 11.85 & 14.55 & 2.60 & 0.06 & 0.57 & 0.09 & 0.13 & 0.53 & 0.11 \\
\hline $2 \mathrm{H}-6,125$ & 13.35 & 17.06 & 2.86 & 0.00 & 0.44 & 0.06 & 0.06 & 0.80 & 0.17 \\
\hline $3 \mathrm{H}-1,125$ & 15.35 & 21.35 & 3.30 & 0.17 & 0.35 & 0.17 & 0.03 & 0.71 & 0.13 \\
\hline $3 \mathrm{H}-2,125$ & 16.85 & 23.55 & 3.53 & 0.09 & 0.43 & 0.05 & 0.14 & 0.50 & 0.05 \\
\hline $3 \mathrm{H}-3,125$ & 18.35 & 25.05 & 3.77 & 0.20 & 0.43 & 0.09 & 0.08 & 0.27 & 0.13 \\
\hline $3 \mathrm{H}-4,125$ & 19.85 & 26.55 & 4.02 & 0.15 & 0.41 & 0.08 & 0.09 & 0.39 & 0.01 \\
\hline $3 \mathrm{H}-5,125$ & 21.35 & 28.05 & 4.26 & 0.22 & 0.48 & 0.18 & 0.10 & 0.56 & 0.09 \\
\hline $3 \mathrm{H}-6,125$ & 22.85 & 29.31 & 4.47 & 0.00 & 0.28 & 0.05 & 0.18 & 0.32 & 0.07 \\
\hline $4 \mathrm{H}-1,125$ & 24.85 & 30.82 & 4.72 & 0.00 & 0.64 & 0.09 & 0.29 & 0.38 & 0.04 \\
\hline $4 \mathrm{H}-2,125$ & 26.35 & 31.95 & 4.91 & 0.15 & 0.34 & 0.19 & 0.03 & 0.36 & 0.07 \\
\hline $4 \mathrm{H}-3,125$ & 27.85 & 33.23 & 5.12 & 0.37 & 0.57 & 0.10 & 0.17 & 0.56 & 0.11 \\
\hline $4 \mathrm{H}-4,125$ & 29.35 & 34.53 & 5.33 & 0.43 & 0.71 & 0.25 & 0.17 & 0.64 & 0.10 \\
\hline $4 \mathrm{H}-5,125$ & 30.85 & 35.99 & 5.57 & 0.14 & 0.41 & 0.08 & 0.15 & 0.49 & 0.09 \\
\hline $4 \mathrm{H}-6,125$ & 32.35 & 37.57 & 5.83 & 0.17 & 0.36 & 0.08 & 0.11 & 0.29 & 0.03 \\
\hline $5 \mathrm{H}-1,125$ & 34.35 & 40.15 & 6.26 & 0.14 & 0.47 & 0.00 & 0.25 & 0.74 & 0.08 \\
\hline $5 \mathrm{H}-2,125$ & 35.85 & 42.06 & 6.58 & 0.19 & 0.35 & 0.00 & 0.19 & 0.43 & 0.06 \\
\hline $5 \mathrm{H}-3,125$ & 37.35 & 44.09 & 6.91 & 0.03 & 0.54 & 0.12 & 0.21 & 0.41 & 0.06 \\
\hline $5 \mathrm{H}-4,125$ & 38.85 & 45.82 & 7.20 & 0.16 & 0.39 & 0.06 & 0.11 & 0.37 & 0.04 \\
\hline $5 \mathrm{H}-5,125$ & 40.35 & 47.49 & 7.42 & 0.20 & 0.44 & 0.09 & 0.09 & 0.48 & 0.13 \\
\hline $5 \mathrm{H}-6,125$ & 41.85 & 49.48 & 7.67 & 0.14 & 0.38 & 0.13 & 0.09 & 0.33 & 0.19 \\
\hline $6 \mathrm{H}-1,125$ & 43.85 & 51.52 & 8.26 & 0.16 & 0.35 & 0.11 & 0.09 & 0.39 & 0.09 \\
\hline $6 \mathrm{H}-2,125$ & 45.35 & 53.30 & 10.67 & 0.04 & 0.28 & 0.06 & 0.03 & 0.29 & 0.06 \\
\hline $6 \mathrm{H}-3,125$ & 46.85 & 54.58 & 12.41 & 0.00 & 0.46 & 0.02 & 0.06 & 0.36 & 0.04 \\
\hline $6 \mathrm{H}-4,125$ & 48.35 & 56.68 & 15.26 & 0.00 & 0.23 & 0.02 & 0.02 & 0.34 & 0.05 \\
\hline $6 \mathrm{H}-5,125$ & 49.85 & 64.22 & 25.47 & 0.65 & 0.14 & 0.02 & 0.01 & 0.18 & 0.02 \\
\hline $6 \mathrm{H}-6,125$ & 51.35 & 69.96 & 33.25 & 0.00 & 1.61 & 0.14 & 0.00 & 1.10 & 0.00 \\
\hline \multicolumn{10}{|l|}{$145-886 \mathrm{~B}-$} \\
\hline $1 \mathrm{H}-1,50$ & 0.50 & 0.77 & 0.14 & 0.00 & 4.35 & 0.00 & 4.26 & 16.43 & 2.90 \\
\hline $1 \mathrm{H}-2,11$ & 1.31 & 2.76 & 0.49 & 0.24 & 3.76 & 0.00 & 4.07 & 12.40 & 2.64 \\
\hline $2 \mathrm{H}-1,50$ & 2.30 & 4.71 & 0.84 & 0.40 & 3.19 & 0.00 & 2.86 & 17.29 & 3.05 \\
\hline $2 \mathrm{H}-2,50$ & 3.80 & 4.76 & 0.85 & 0.40 & 6.33 & 0.00 & 4.41 & 16.71 & 3.21 \\
\hline $2 \mathrm{H}-3,50$ & 5.30 & 4.82 & 0.86 & 0.52 & 4.54 & 0.00 & 3.32 & 17.24 & 2.39 \\
\hline $2 \mathrm{H}-4,50$ & 6.80 & 4.87 & 0.87 & 0.00 & 2.75 & 0.00 & 1.97 & 11.26 & 1.82 \\
\hline $2 \mathrm{H}-5,50$ & 8.30 & 4.93 & 0.88 & 0.72 & 5.37 & 0.00 & 3.66 & 16.52 & 3.31 \\
\hline $2 \mathrm{H}-6,50$ & 9.80 & 4.98 & 0.89 & 0.79 & 4.50 & 0.00 & 4.58 & 16.73 & 2.71 \\
\hline $2 \mathrm{H}-7,45$ & 11.25 & 7.49 & 1.34 & 0.11 & 2.37 & 0.00 & 2.15 & 10.69 & 2.17 \\
\hline $3 \mathrm{H}-1,50$ & 11.80 & 9.11 & 1.63 & 1.70 & 6.95 & 0.00 & 5.41 & 25.88 & 5.16 \\
\hline $3 \mathrm{H}-2,50$ & 13.30 & 10.60 & 1.89 & 0.00 & 2.68 & 0.00 & 1.37 & 9.87 & 3.76 \\
\hline $3 \mathrm{H}-3,50$ & 14.80 & 12.61 & 2.25 & 0.09 & 4.57 & 0.00 & 2.09 & 12.85 & 2.61 \\
\hline $3 \mathrm{H}-4,50$ & 16.30 & 14.65 & 2.61 & 0.29 & 4.97 & 0.00 & 2.60 & 18.48 & 2.10 \\
\hline $3 \mathrm{H}-5,50$ & 17.80 & 16.15 & 2.76 & 0.00 & 3.79 & 0.00 & 2.60 & 11.45 & 2.19 \\
\hline $3 \mathrm{H}-6,50$ & 19.30 & 17.71 & 2.93 & 0.00 & 3.72 & 0.30 & 2.42 & 11.72 & 2.40 \\
\hline $4 \mathrm{H}-1,50$ & 21.30 & 20.47 & 3.21 & 0.70 & 5.31 & 0.49 & 2.37 & 13.19 & 2.11 \\
\hline $4 \mathrm{H}-2,50$ & 22.80 & 22.55 & 3.43 & 0.01 & 3.30 & 0.00 & 1.64 & 13.82 & 2.33 \\
\hline $4 \mathrm{H}-3,50$ & 24.30 & 24.59 & 3.69 & 1.06 & 5.96 & 0.77 & 2.34 & 14.59 & 1.87 \\
\hline $4 \mathrm{H}-4,50$ & 25,80 & 26.17 & 3.95 & 0.00 & 4.61 & 0.71 & 2.21 & 10.77 & 2.22 \\
\hline $4 \mathrm{H}-5,50$ & 27.30 & 27.52 & 4.18 & 1.79 & 10.99 & 1.95 & 5.70 & 16.84 & 3.07 \\
\hline $4 \mathrm{H}-6,50$ & 28.80 & 28.87 & 4.40 & 0.00 & 4.81 & 0.00 & 4.07 & 9.92 & 1.76 \\
\hline $5 \mathrm{H}-1,50$ & 30.80 & 30.66 & 4.69 & 0.20 & 4.70 & 0.10 & 2.54 & 9.84 & 2.13 \\
\hline $5 \mathrm{H}-2,50$ & 32.30 & 32.01 & 4.92 & 1.54 & 3.77 & 0.00 & 1.89 & 8.97 & 4.57 \\
\hline $5 \mathrm{H}-3,50$ & 33.80 & 33.50 & 5.16 & 0.18 & 3.18 & 0.00 & 2.09 & 13.50 & 2.49 \\
\hline $5 \mathrm{H}-4,50$ & 35.30 & 35.00 & 5.41 & 1.56 & 5.26 & 0.00 & 4.48 & 16.17 & 3.23 \\
\hline $5 \mathrm{H}-5,50$ & 36.80 & 36.50 & 5.66 & 0.70 & 6.82 & 1.30 & 2.13 & 13.91 & 2.25 \\
\hline $5 \mathrm{H}-6,50$ & 38.30 & 38.00 & 5.91 & 0.00 & 3.56 & 0.00 & 1.87 & 9.95 & 2.26 \\
\hline $5 \mathrm{H}-7,50$ & 39.80 & 39.56 & 6.16 & 0.74 & 6.68 & 1.21 & 2.63 & 18.63 & 2.67 \\
\hline $6 \mathrm{H}-1,50$ & 40.30 & 40.39 & 6.30 & 0.02 & 5.01 & 1.21 & 1.63 & 13.33 & 2.25 \\
\hline $6 \mathrm{H}-2,50$ & 41.80 & 41.95 & 6.56 & 0.00 & 3.32 & 0.00 & 2.49 & 12.47 & 2.19 \\
\hline $6 \mathrm{H}-3,50$ & 43.30 & 43.45 & 6.81 & 0.08 & 4.21 & 0.00 & 3.25 & 13.77 & 2.10 \\
\hline $6 \mathrm{H}-4,50$ & 44.80 & 44.95 & 7.05 & 0.00 & 3.40 & 0.32 & 2.04 & 13.36 & 2.43 \\
\hline $6 \mathrm{H}-5,50$ & 46.30 & 46.45 & 7.30 & 0.24 & 4.82 & 0.44 & 1.65 & 14.80 & 1.92 \\
\hline $6 \mathrm{H}-6,52$ & 47.82 & 48.12 & 7.58 & 0.00 & 2.60 & 0.71 & 0.81 & 11.48 & 1.25 \\
\hline $6 \mathrm{H}-7,50$ & 49.30 & 50.28 & 7.93 & 0.00 & 4.74 & 0.77 & 1.31 & 15.66 & 2.21 \\
\hline $7 \mathrm{H}-1,47$ & 49.77 & 50.97 & 8.05 & 0.00 & 1.65 & 0.25 & 0.70 & 9.89 & 1.29 \\
\hline $7 \mathrm{H}-2,47$ & 51.27 & 53.02 & 8.39 & 0.00 & 2.49 & 0.56 & 0.39 & 11.63 & 1.61 \\
\hline $7 \mathrm{H}-3,47$ & 52.77 & 54.52 & 8.63 & 0.00 & 0.88 & 0.00 & 0.00 & 7.61 & 3.20 \\
\hline $7 \mathrm{H}-4,47$ & 54.27 & 55.55 & 8.80 & 0.11 & 1.53 & 0.00 & 0.00 & 10.39 & 1.83 \\
\hline $7 \mathrm{H}-5,47$ & 55.77 & 57.20 & 9.08 & 0.53 & 2.78 & 0.00 & 0.00 & 13.35 & 2.26 \\
\hline $7 \mathrm{H}-6,47$ & 57.27 & 58.79 & 9.34 & 0.04 & 0.88 & 0.00 & 4.04 & 7.61 & 3.20 \\
\hline $7 \mathrm{H}-7,47$ & 58.77 & 60.22 & 9.57 & 1.83 & 0.95 & 0.00 & 0.00 & 7.66 & 2.78 \\
\hline $8 X-1,50$ & 59.30 & 60.75 & 9.66 & 0.00 & 0.82 & 0.00 & 0.00 & 1.43 & 0.24 \\
\hline
\end{tabular}

Note: Peak areas normalized to internal standards. 
Table 5. Normalized mineral peak areas for the 2-20 $\mu \mathrm{m}$ fraction of Holes $885 \mathrm{~A}$ and 886B.

\begin{tabular}{|c|c|c|c|c|c|c|c|c|c|}
\hline $\begin{array}{l}\text { Core, section, } \\
\text { interval }(\mathrm{cm})\end{array}$ & $\begin{array}{l}\text { Depth } \\
\text { (mbsf) }\end{array}$ & $\begin{array}{l}\text { Composite } \\
\text { depth }(\mathrm{m})\end{array}$ & $\begin{array}{l}\text { Age } \\
\text { (Ma) }\end{array}$ & $\begin{array}{l}\text { Smectite } \\
\text { peak area }\end{array}$ & $\begin{array}{c}\text { Illite } \\
\text { peak area }\end{array}$ & $\begin{array}{l}\text { Kaolinite } \\
\text { peak area }\end{array}$ & $\begin{array}{c}\text { Chlorite } \\
\text { peak area }\end{array}$ & $\begin{array}{c}\text { Quartz } \\
\text { peak area }\end{array}$ & $\begin{array}{c}\text { Plagioclase } \\
\text { peak area }\end{array}$ \\
\hline \multicolumn{10}{|l|}{$145-885 \mathrm{~A}-$} \\
\hline $1 \mathrm{H}-1,25$ & 0.25 & 0.30 & 0.05 & 0.35 & 2.12 & 0.00 & 1.58 & 15.50 & 0.60 \\
\hline $1 \mathrm{H}-1,125$ & 1.25 & 1.65 & 0.26 & 0.52 & 2.98 & 0.00 & 2.26 & 23.35 & 3.57 \\
\hline $1 \mathrm{H}-2,25$ & 1.75 & 2.34 & 0.37 & 0.54 & 3.21 & 0.00 & 1.34 & 17.03 & 2.95 \\
\hline IH $-2,125$ & 2.75 & 3.89 & 0.62 & 0.09 & 1.44 & 0.00 & 0.74 & 7.79 & 1.46 \\
\hline $1 \mathrm{H}-3,25$ & 3.25 & 4.34 & 0.69 & 0.28 & 1.49 & 0.00 & 1.31 & 11.06 & 2.06 \\
\hline $1 \mathrm{H}-3,125$ & 4.25 & 5.16 & 0.85 & 0.00 & 2.45 & 0.00 & 1.82 & 15.41 & 2.88 \\
\hline $2 \mathrm{H}-1,25$ & 4.85 & 5.63 & 0.96 & 0.10 & 3.14 & 0.00 & 1.65 & 16.49 & 2.95 \\
\hline $2 \mathrm{H}-1,125$ & 5.85 & 6.72 & 1.11 & 0.00 & 2.27 & 0.00 & 1.38 & 12.01 & 2.73 \\
\hline $2 \mathrm{H}-2,25$ & 6.35 & 7.24 & 1.27 & 0.00 & 2.71 & 0.00 & 1.30 & 13.11 & 2.26 \\
\hline $2 \mathrm{H}-2,125$ & 7.35 & 8.19 & 1.48 & 0.00 & 1.89 & 0.00 & 0.99 & 11.13 & 2.10 \\
\hline $2 \mathrm{H}-3,25$ & 7.85 & 8.63 & 1.57 & 0.32 & 2.00 & 0.00 & 0.00 & 9.63 & 3.01 \\
\hline $2 \mathrm{H}-3,125$ & 8.85 & 9.61 & 1.78 & 0.00 & 2.75 & 0.00 & 1.13 & 13.36 & 2.46 \\
\hline $2 \mathrm{H}-4,125$ & 10.35 & 12.12 & 2.23 & 0.26 & 3.04 & 0.00 & 1.39 & 18.31 & 2.56 \\
\hline $2 \mathrm{H}-5,125$ & 11.85 & 14.55 & 2.60 & 0.00 & 2.10 & 0.00 & 0.84 & 13.29 & 2.24 \\
\hline $2 \mathrm{H}-6,125$ & 13.35 & 17.06 & 2.86 & 0.00 & 1.46 & 0.00 & 0.00 & 8.54 & 3.59 \\
\hline $3 \mathrm{H}-1,125$ & 15.35 & 21.35 & 3.30 & 1.44 & 2.79 & 0.00 & 1.69 & 12.88 & 2.47 \\
\hline $3 \mathrm{H}-2,125$ & 16.85 & 23.55 & 3.53 & 0.46 & 3.13 & 0.00 & 1.54 & 9.98 & 1.69 \\
\hline $3 \mathrm{H}-3,125$ & 18.35 & 25.05 & 3.77 & 1.57 & 9.53 & 0.00 & 4.00 & 15.46 & 5.71 \\
\hline $3 \mathrm{H}-4,125$ & 19.85 & 26.55 & 4.02 & 1.96 & 11.59 & 1.04 & 5.45 & 17.12 & 3.45 \\
\hline $3 \mathrm{H}-5,125$ & 21.35 & 28.05 & 4.26 & 1.43 & 6.40 & 1.14 & 3.20 & 14.36 & 2.34 \\
\hline $3 \mathrm{H}-6,125$ & 22.85 & 29.31 & 4.47 & 1.69 & 5.65 & 0.00 & 3.61 & 8.97 & 1.86 \\
\hline $4 \mathrm{H}-1,125$ & 24.85 & 30.82 & 4.72 & 4.54 & 22.16 & 2.30 & 11.66 & 14.14 & 2.84 \\
\hline $4 \mathrm{H}-2,125$ & 26.35 & 31.95 & 4.91 & 3.13 & 9.26 & 0.00 & 7.14 & 19.03 & 4.16 \\
\hline $4 \mathrm{H}-3,125$ & 27.85 & 33.23 & 5.12 & 1.14 & 3.70 & 0.32 & 2.05 & 20.86 & 2.11 \\
\hline $4 \mathrm{H}-4,125$ & 29.35 & 34.53 & 5.33 & 0.68 & 7.23 & 0.45 & 3.46 & 25.46 & 3.25 \\
\hline $4 \mathrm{H}-5,125$ & 30.85 & 35.99 & 5.57 & 0.93 & 2.84 & 0.00 & 1.15 & 11.57 & 1.94 \\
\hline $4 \mathrm{H}-6,125$ & 32.35 & 37.57 & 5.83 & 0.74 & 4.14 & 0.26 & 2.00 & 13.18 & 1.88 \\
\hline $5 \mathrm{H}-1,125$ & 34.35 & 40.15 & 6.26 & 0.29 & 2.90 & 0.00 & 1.91 & 12.98 & 1.97 \\
\hline $5 \mathrm{H}-2,125$ & 35.85 & 42.06 & 6.58 & 0.03 & 4.05 & 0.00 & 2.26 & 15.30 & 3.04 \\
\hline $5 \mathrm{H}-3,125$ & 37.35 & 44.09 & 6.91 & 1.10 & 6.48 & 1.06 & 3.58 & 12.86 & 1.66 \\
\hline $5 \mathrm{H}-4,125$ & 38.85 & 45.82 & 7.20 & 0.00 & 3.32 & 0.51 & 1.43 & 13.77 & 1.85 \\
\hline $5 \mathrm{H}-5,125$ & 40.35 & 47.49 & 7.42 & 0.00 & 3.18 & 0.00 & 1.40 & 15.35 & 2.48 \\
\hline $5 \mathrm{H}-6,125$ & 41.85 & 49.48 & 7.67 & 0.08 & 2.80 & 0.00 & 1.36 & 10.17 & 1.51 \\
\hline $6 \mathrm{H}-1,125$ & 43.85 & 51.52 & 8.26 & 0.00 & 2.60 & 0.51 & 0.78 & 11.94 & 2.02 \\
\hline $6 \mathrm{H}-2,125$ & 45.35 & 53.30 & 10.67 & 0.52 & 2.61 & 0.28 & 0.50 & 12.65 & 2.06 \\
\hline $6 \mathrm{H}-3,125$ & 46.85 & 54.58 & 12.41 & 0.00 & 2.78 & 0.34 & 0.00 & 10.76 & 2.04 \\
\hline $6 \mathrm{H}-4,125$ & 48.35 & 56.68 & 15.26 & 0.64 & 3.13 & 0.00 & 0.00 & 14.13 & 2.71 \\
\hline $6 \mathrm{H}-5,125$ & 49.85 & 64.22 & 25.47 & 1.38 & 2.46 & 0.00 & 0.34 & 13.11 & 0.81 \\
\hline $6 \mathrm{H}-6,125$ & 51.35 & 69.96 & 33.25 & 0.00 & 2.49 & 0.38 & 0.00 & 1.35 & 0.25 \\
\hline \multicolumn{10}{|l|}{ 145-886B- } \\
\hline $1 \mathrm{H}-1,50$ & 0.50 & 0.77 & 0.14 & 0.00 & 4.35 & 0.00 & 4.26 & 16.43 & 2.90 \\
\hline $1 \mathrm{H}-2,11$ & 1.31 & 2.76 & 0.49 & 0.24 & 3.76 & 0.00 & 4.07 & 12.40 & 2.64 \\
\hline $2 \mathrm{H}-1,50$ & 2.30 & 4.71 & 0.84 & 0.40 & 3.19 & 0.00 & 2.86 & 17.29 & 3.05 \\
\hline $2 \mathrm{H}-2,50$ & 3.80 & 4.76 & 0.85 & 0.40 & 6.33 & 0.00 & 4.41 & 16.71 & 3.21 \\
\hline $2 \mathrm{H}-3,50$ & 5.30 & 4.82 & 0.86 & 0.52 & 4.54 & 0.00 & 3.32 & 17.24 & 2.39 \\
\hline $2 \mathrm{H}-4,50$ & 6.80 & 4.87 & 0.87 & 0.00 & 2.75 & 0.00 & 1.97 & 11.26 & 1.82 \\
\hline $2 \mathrm{H}-5,50$ & 8.30 & 4.93 & 0.88 & 0.72 & 5.37 & 0.00 & 3.66 & 16.52 & 3.31 \\
\hline $2 \mathrm{H}-6,50$ & 9.80 & 4.98 & 0.89 & 0.79 & 4.50 & 0.00 & 4.58 & 16.73 & 2.71 \\
\hline $2 \mathrm{H}-7,45$ & 11.25 & 7.49 & 1.34 & 0.11 & 2.37 & 0.00 & 2.15 & 10.69 & 2.17 \\
\hline $3 \mathrm{H}-1,50$ & 11.80 & 9.11 & 1.63 & 1.70 & 6.95 & 0.00 & 5.41 & 25.88 & 5.16 \\
\hline $3 \mathrm{H}-2,50$ & 13.30 & 10.60 & 1.89 & 0.00 & 2.68 & 0.00 & 1.37 & 9.87 & 3.76 \\
\hline $3 \mathrm{H}-3,50$ & 14.80 & 12.61 & 2.25 & 0.09 & 4.57 & 0.00 & 2.09 & 12.85 & 2.61 \\
\hline $3 \mathrm{H}-4,50$ & 16.30 & 14.65 & 2.61 & 0.29 & 4.97 & 0.00 & 2.60 & 18.48 & 2.10 \\
\hline $3 \mathrm{H}-5,50$ & 17.80 & 16.15 & 2.76 & 0.00 & 3.79 & 0.00 & 2.60 & 11.45 & 2.19 \\
\hline $3 \mathrm{H}-6,50$ & 19.30 & 17.71 & 2.93 & 0.00 & 3.72 & 0.30 & 2.42 & 11.72 & 2.40 \\
\hline $4 \mathrm{H}-1,50$ & 21.30 & 20.47 & 3.21 & 0.70 & 5.31 & 0.49 & 2.37 & 13.19 & 2.11 \\
\hline $4 \mathrm{H}-2,50$ & 22.80 & 22.55 & 3.43 & 0.01 & 3.30 & 0.00 & 1.64 & 13.82 & 2.33 \\
\hline $4 \mathrm{H}-3,50$ & 24.30 & 24.59 & 3.69 & 1.06 & 5.96 & 0.77 & 2.34 & 14.59 & 1.87 \\
\hline $4 \mathrm{H}-4,50$ & 25.80 & 26.17 & 3.95 & 0.00 & 4.61 & 0.71 & 2.21 & 10.77 & 2.22 \\
\hline $4 \mathrm{H}-5,50$ & 27.30 & 27.52 & 4.18 & 1.79 & 10.99 & 1.95 & 5.70 & 16.84 & 3.07 \\
\hline $4 \mathrm{H}-6,50$ & 28.80 & 28.87 & 4.40 & 0.00 & 4.81 & 0.00 & 4.07 & 9.92 & 1.76 \\
\hline $5 \mathrm{H}-1,50$ & 30.80 & 30.66 & 4.69 & 0.20 & 4.70 & 0.10 & 2.54 & 9.84 & 2.13 \\
\hline $5 \mathrm{H}-2,50$ & 32.30 & 32.01 & 4.92 & 1.54 & 3.77 & 0.00 & 1.89 & 8.97 & 4.57 \\
\hline $5 \mathrm{H}-3,50$ & 33.80 & 33.50 & 5.16 & 0.18 & 3.18 & 0.00 & 2.09 & 13.50 & 2.49 \\
\hline $5 \mathrm{H}-4,50$ & 35.30 & 35.00 & 5.41 & 1.56 & 5.26 & 0.00 & 4.48 & 16.17 & 3.23 \\
\hline $5 \mathrm{H}-5,50$ & 36.80 & 36.50 & 5.66 & 0.70 & 6.82 & 1.30 & 2.13 & 13.91 & 2.25 \\
\hline $5 \mathrm{H}-6,50$ & 38.30 & 38.00 & 5.91 & 0.00 & 3.56 & 0.00 & 1.87 & 9.95 & 2.26 \\
\hline $5 \mathrm{H}-7,50$ & 39.80 & 39.56 & 6,16 & 0.74 & 6.68 & 1.21 & 2.63 & 18.63 & 2.67 \\
\hline $6 \mathrm{H}-\mathrm{I}, 50$ & 40.30 & 40.39 & 6.30 & 0.02 & 5.01 & 1.21 & 1.63 & 13.33 & 2.25 \\
\hline $6 \mathrm{H}-2,50$ & 41.80 & 41.95 & 6.56 & 0.00 & 3.32 & 0.00 & 2.49 & 12.47 & 2.19 \\
\hline $6 \mathrm{H}-3,50$ & 43.30 & 43.45 & 6.81 & 0.08 & 4.21 & 0.00 & 3.25 & 13.77 & 2.10 \\
\hline $6 \mathrm{H}-4,50$ & 44.80 & 44.95 & 7.05 & 0.00 & 3.40 & 0.32 & 2.04 & 13.36 & 2.43 \\
\hline $6 \mathrm{H}-5,50$ & 46.30 & 46.45 & 7.30 & 0.24 & 4.82 & 0.44 & 1.65 & 14.80 & 1.92 \\
\hline $6 \mathrm{H}-6,52$ & 47.82 & 48.12 & 7.58 & 0.00 & 2.60 & 0.71 & 0.81 & 11.48 & 1.25 \\
\hline $6 \mathrm{H}-7,50$ & 49.30 & 50.28 & 7.93 & 0.00 & 4.74 & 0.77 & 1.31 & 15.66 & 2.21 \\
\hline $7 \mathrm{H}-1,47$ & 49.77 & 50.97 & 8.05 & 0.00 & 1.65 & 0.25 & 0.70 & 9.89 & 1.29 \\
\hline $7 \mathrm{H}-2,47$ & 51.27 & 53.02 & 8.39 & 0.00 & 2.49 & 0.56 & 0.39 & 11.63 & 1.61 \\
\hline $7 \mathrm{H}-3,47$ & 52.77 & 54.52 & 8.63 & 0.00 & 0.88 & 0.00 & 0.00 & 7.61 & 3.20 \\
\hline $7 \mathrm{H}-4,47$ & 54.27 & 55.55 & 8.80 & 0.11 & 1.53 & 0.00 & 0.00 & 10.39 & 1.83 \\
\hline $7 \mathrm{H}-5,47$ & 55.77 & 57.20 & 9.08 & 0.53 & 2.78 & 0.00 & 0.00 & 13.35 & 2.26 \\
\hline $7 \mathrm{H}-6,47$ & 57.27 & 58.79 & 9.34 & 0.04 & 0.88 & 0.00 & 4.04 & 7.61 & 3.20 \\
\hline $7 \mathrm{H}-7,47$ & 58.77 & 60.22 & 9.57 & 1.83 & 0.95 & 0.00 & 0.00 & 7.66 & 2.78 \\
\hline $8 X-1,50$ & 59.30 & 60.75 & 9.66 & 0.00 & 0.82 & 0.00 & 0.00 & 1.43 & 0.24 \\
\hline
\end{tabular}

Note: Peak areas normalized to internal standards. 
genically in the relatively thin sediments near Holes $885 \mathrm{~A}$ and $886 \mathrm{~B}$. For both size fractions, there is a relative increase in illite weight percent centered on about 30 med (Figs. 2B, 3B). The magnitude of the increase is largest in the $2-20 \mu \mathrm{m}$ size fraction and smallest in the $<2 \mu \mathrm{m}$ size fraction. The $<2 \mu \mathrm{m}$ size fraction demonstrates the greatest variability in illite throughout all depths, and there is some suggestion of an illite increase in the upper $10 \mathrm{~m}$ of the Unit I red clay. The $2-20 \mu \mathrm{m}$ size fraction has uniform and approximately equal illite concentrations in the upper and lower clay units.

\section{Kaolinite}

Kaolinite is a terrigenous weathering product usually associated with strong hydrolysis. The error associated with kaolinite determination in this study is large due to the generally low kaolinite concentration in this study area, so only general comments may be made about variations in this mineral group. Kaolinite is present in highly variable amounts in all units for the $<2 \mu \mathrm{m}$ size fraction (Fig. 2C). The 2-20 $\mu \mathrm{m}$ size fraction contains a significant, but highly variable amount of kaolinite in only the diatom ooze unit (Fig. 3C).

\section{Chlorite}

Chlorite is a mineral phase that is usually used to indicate mechanical weathering of terrigenous sediments as it is highly susceptible to hydrolysis. The $<2 \mu \mathrm{m}$ fraction (Fig. 2D) shows low variability and low concentrations in the lower brown clay unit and high variability in the diatom unit and the upper red clay unit. Chlorite concentrations increase in sediments shallower than $10 \mathrm{mcd}$. The chlorite concentration in the 2-20 $\mu \mathrm{m}$ size fraction (Fig. 3D) is low in the lower brown clay unit, and high and variable in the diatom ooze, where again a concentration peak is observed centered at about $30 \mathrm{mcd}$. The concentration of chlorite drops at the Unit I/II boundary, and the Hole 885A and $886 \mathrm{~B}$ records diverge; the normalized peak areas are lower in Hole $885 \mathrm{~A}$ than in Hole $886 \mathrm{~B}$. This divergence appears to be a systematic laboratory error.

\section{Quartz}

Quartz is a primary mineral that is not formed authigenically on the seafloor. Both records (Figs. 2E, 3E) show a high variability, but uniform proportion, of quartz throughout all units. Quartz concentrations seem to increase in sediments shallower than $10 \mathrm{mcd}$ in the $<2$ $\mu \mathrm{m}$ fraction.

\section{Plagioclase}

Plagioclase is also a primary mineral not authigenically formed on the seafloor. Figures $2 \mathrm{~F}$ and $3 \mathrm{~F}$ illustrate the plagioclase distribution in the Hole $885 \mathrm{~A}$ and $886 \mathrm{~B}$ sediments. Both records are variable throughout all units. The $<2 \mu \mathrm{m}$ fraction shows a slight upcore increase, while the $2-20 \mu \mathrm{m}$ fraction is relatively uniform in concentration.

\section{Peak Height Ratios}

Another way to examine the mineralogy data is to look at relative change between mineral groups. The compositional changes between groups of minerals are indicative of either changing tectonics, climatic conditions, source area, or diagenetic processes. Figures 4 and 5 illustrate peak area ratios between pairs of minerals for the $<2 \mu \mathrm{m}$ and $2-20 \mu \mathrm{m}$ size fractions. The ratio of kaolinite to illite varies as a function of the amount of source area hydrolysis, which is related to rainfall. Kaolinite formation is favored in temperate, well-drained soils, whereas illite is a ubiquitous clay mineral associated with cooler, drier climates. The $<2 \mu \mathrm{m}$ fraction is highly variable throughout sediments from both Holes $885 \mathrm{~A}$ and $886 \mathrm{~B}$ (Fig. 4A). The 2-20 $\mu \mathrm{m}$ size fraction (Fig. 5A) suggests an abrupt compositional change at the Unit II/III boundary. Unit III contains no kaolinite in this size fraction.
There is a sharp increase to an overall maximum in the proportion of kaolinite at the bottom of Unit II, followed by a monotonic decline that reaches zero again at the base of Unit I.

The ratio of smectite/illite (S/I) is usually indicative of either volcanism or hydrolysis in deep-sea sediments. However, in this case (Figs. 4B, 5B), the large $\mathrm{S} / \mathrm{I}$ ratios in Unit III is consistent with a large hydrothermal component in the lower brown clay unit (Dickens and Owen, this volume). The S/I ratio drops at the base of Unit II and remains relatively constant throughout the remainder of the sediment column in the $<2 \mu \mathrm{m}$ and $2-20 \mu \mathrm{m}$ fraction.

Kaolinite to quartz (Figs. 4C, 5C) is the ratio of a hydrolysis sensitive mineral to a primary mineral. In both size fractions, K/Q is generally low in Unit III, reaches a maximum at about $30 \mathrm{mcd}$, and decreases toward the top of the core. This variation is more pronounced in the $2-20 \mu \mathrm{m}$ size fraction, where $\mathrm{K} / \mathrm{Q}$ goes to zero at the Unit I/II boundary.

The illite/quartz (Figs. 4D, 5D) ratios display decreasing values in the $<2 \mu \mathrm{m}$ fraction from the bottom to the top of Unit III; I/Q is relatively uniform in the $2-20 \mu \mathrm{m}$ fraction of this interval. In both size fractions, the lower part of Unit II displays uniform ratios; there is a peak in this ratio centered at about 30 mcd. This peak is much more pronounced in the $2-20 \mu \mathrm{m}$ size fraction. The ratio decreases slightly in both size fractions from the bottom to the top of the upper red clay unit.

In both size fractions, chlorite/quartz ratios (Figs. 4E, 5E), indicative of mechanical weathering, are generally very small in lower clay Unit III, show a slight increase at the Unit II/III boundary, and another small step up at $\sim 45 \mathrm{mcd}$. There is a sharp peak in this ratio centered at approximately $30 \mathrm{mcd}$, followed by uniform values throughout the remainder of the diatom unit and Unit $\mathrm{L}$.

\section{Rock Magnetics vs. Mineralogy}

Finally, we compare the rock-magnetic properties to the mineralogy, to examine how the two covary. Figure 6 illustrates the lowfrequency susceptibility and the $<2 \mu \mathrm{m}$ quartz peak area plotted vs. composite depth. The two variables covary in the upper red clay and diatom ooze units. However, the signals decouple at the Unit II/III boundary. This decoupling of the signal implies that the sedimentary material in Unit III is from a different source than the terrigenous material in Unit I.

Figure 7 illustrates the illite/quartz ratio and the S-ratio plotted as a function of composite depth. These variables strongly correspond at about $30 \mathrm{mcd}$. Illite/quartz increases occur at the same place that the rock magnetic parameter, $\mathrm{S}$, indicates a shift from low to high coercivity iron oxide mineralogy.

\section{Accumulation Rates}

Figures illustrating the mass accumulation rates (MARs) of different parameters over time are useful here because the diatom ooze represents a large dilutional component to the terrigenous sediments proximal to Sites 885 and 886 . Figure 8 illustrates the MARs of the bulk sediment, terrigenous fraction, and the $2-20 \mu \mathrm{m}$ and $<2 \mu \mathrm{m}$ fractions of the sediment used for mineralogical analysis. There is offset in the terrigenous MARs of Holes $885 \mathrm{~A}$ and $886 \mathrm{~B}$ in the upper clay unit. This could be the result of either systematic laboratory error, sediment winnowing, or focusing differences between the two sites. Because there are no systematic offsets in the primary rock magnetic parameters, or in the bulk MARs, we think the former is the case. Snoeckx et al. (this volume) provide a detailed interpretation of the eolian accumulation at Sites 885 and 886 . For the parameters presented, we display only the upper two units for the mass accumulation discussion; we have few samples from the lower clay unit, and the ages of these sediments are uncertain.

Figure 9 displays the magnetic concentration parameters plotted as accumulation rates vs. age. The magnetic-iron oxide concentration parameters $\left(X_{l f}\right.$ and $\left.H I R M\right)$ track the terrigenous MARs. These 

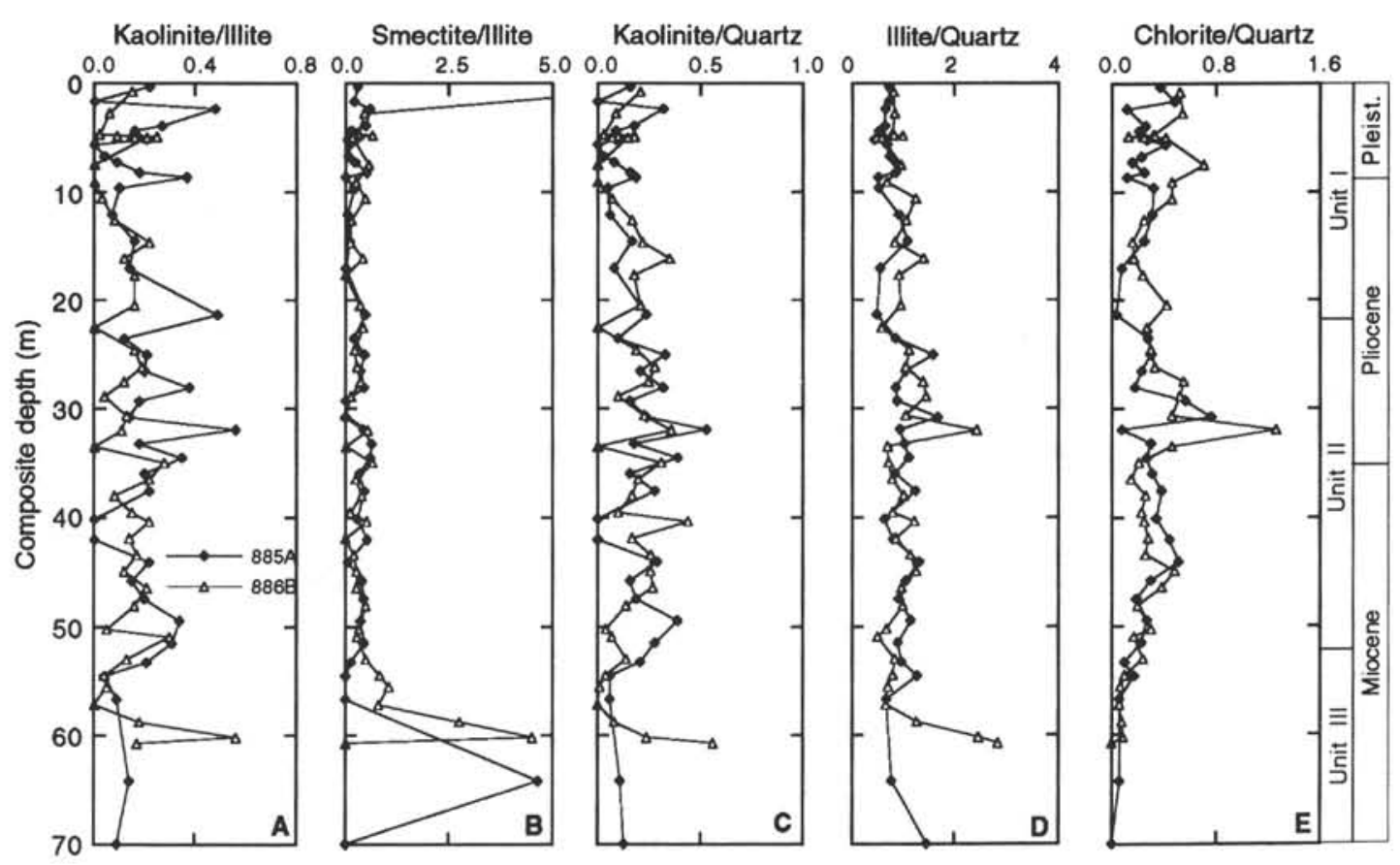

Figure 4. Peak area ratios for the $<2 \mu \mathrm{m}$ terrigenous size fraction vs. composite depth for Holes $885 \mathrm{~A}$ and $886 \mathrm{~B}$.
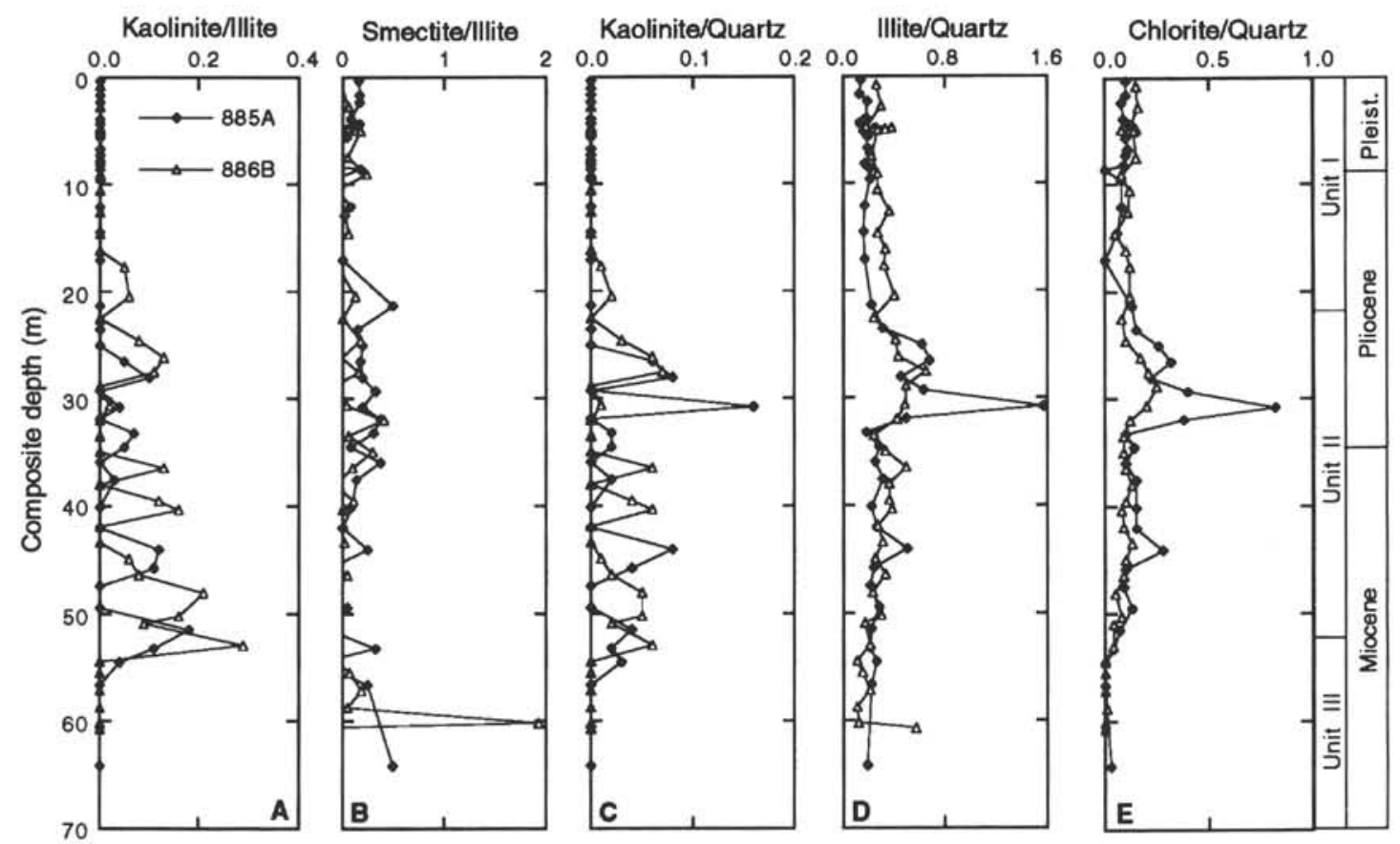

Figure 5. Peak area ratios for the $2-20 \mu \mathrm{m}$ terrigenous size fraction vs. composite depth for Holes $885 \mathrm{~A}$ and $886 \mathrm{~B}$.

records show a small, LSR-controlled peak at the base of Unit II $(\sim 7.5$ $\mathrm{Ma})$, low accumulation and low variability through the middle part of this unit, followed by an abrupt increase in accumulation at $\sim 3.8$ $\mathrm{Ma}$, which continues through all of Unit I.

Figure 10 illustrates the accumulation rates for the mineral phases identified in the $<2 \mu \mathrm{m}$ size fraction. With the exception of kaolinite, which increases in the Unit II diatom ooze in the $2-20 \mu \mathrm{m}$ size fraction, the $<2 \mu \mathrm{m}$ patterns are identical with the larger size class, and are not illustrated. For all minerals, the accumulation rate has small peaks at the base of Unit II at $\sim 7.5,6.5$, and $5.1 \mathrm{Ma}$ and decreases to low values after $5 \mathrm{Ma}$. The MARs of the minerals stay low until an abrupt increase at about $3.8 \mathrm{Ma}$, which continues to increase throughout the late Pleistocene.

\section{DISCUSSION}

There are several changes in the mineralogy and magnetic properties that imply varying environmental conditions at Sites 885 and 886. Unit III, the lower brown clay unit, is characterized by high concentrations of fine-grained ferrimagnetic iron oxides, that contain a softer magnetic carrier than the Unit I sediments, low bulk and terrigenous MARs, and a mineralogy characterized by a large proportion of smec- 


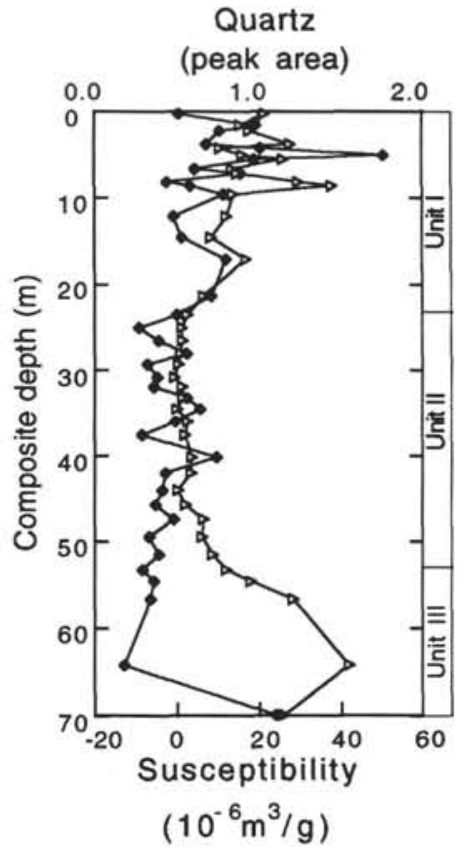

Figure 6 . Less than $2 \mu \mathrm{m}$ quartz peak area and low frequency susceptibility vs. composite depth for Hole 885A. Open symbols = susceptibility, solid symbols $=$ quartz peak area.

tite relative to the other units. Unit II, the diatom ooze, is marked by low magnetic-iron oxide concentrations and fine grain sizes, moderate sedimentation rates, low bulk and terrigenous MARs, and an interval of anomalous mineralogy and magnetic composition centered at $30 \mathrm{mcd}$.

The Unit I/II boundary is marked by a step up in the terrigenous MAR and magnetic-iron oxide concentrations. These increases continue to the top of the core and are accompanied by an increase in the iron oxide grain size, as well as an increase in the chlorite concentrations.

\section{Sediment Sources}

The potentially important sediment sources proximal to Site 885 and 886 now and in the past include eolian terrigenous and volcanogenic sediment, siliceous biogenic material, and hydrothermal precipitates. The rock-magnetic and mineralogy measurements mimic the major lithological changes, which account for most of the variation observed for these parameters. There is close covariance of the mineralogy and magnetics in the upper two lithologic units (Fig. 6). The decoupling of the signal at the base of Unit II, specifically a drop in quartz concentration together with a sharp increase in the iron oxide concentration, occurs at the same time as a shift toward a magnetic composition enriched (relative to the Unit I sediments) in a low-coercivity component. Large concentrations of iron oxides in deep-sea sediments are produced by hydrothermal, terrigenous sedimentary or volcanogenic processes. The sediment in Unit III is likely composed of a large hydrothermal component (Dickens and Owen, this volume). Hydrothermal sediment is characterized by a wide variety of magnetic carriers, such as magnetite, hematite or goethite, which were observed in shipboard smear-slide analyses (Rea, Basov, Janecek, Palmer-Julson, et al., 1993).

Although the magnetic composition of terrigenous material would depend on the source area composition, terrigenous material from a volcanogenic environment would be enriched in low-coercivity magnetic minerals. We hypothesize that the hydrothermal sediments in Unit III may also contain a small amount of eolian, andesitic, terrigenous material. Kyte et al. (1993) propose andesitic volcanism in western Mexico as a source for the Late Cretaceous terrigenous sediments identified in the bottom of Core LL44-GPC3. Plate tec-

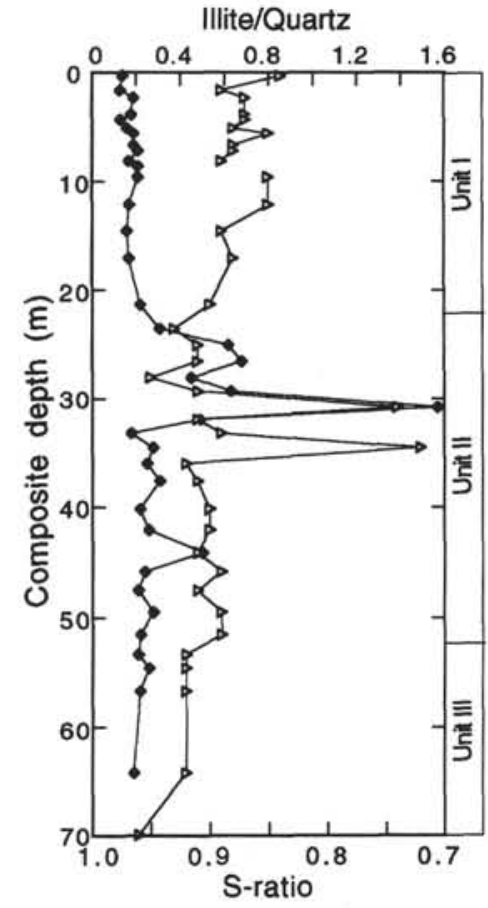

Figure 7. The S-ratio and $2-20 \mu \mathrm{m}$ illite/quartz ratio vs. composite depth for Hole $885 \mathrm{~A}$. Open symbols $=\mathrm{S}$-ratio, solid symbols $=$ illite/quartz peak area .

tonic calculations indicate that conditions may have been favorable for some andesitic eolian contribution in the past. The clay material in the top two units of the sediment column is likely from an Asian source, as indicated by the present location of the site and the covariance of the rock-magnetic and mineralogy measurements. The increase in the importance of this eolian component is considered in the context of the climate and tectonic forces driving the signal.

\section{Tectonic and Climatic Variation}

There are two types of tectonic variation that we need to consider to interpret the mineralogical and rock magnetic signals from Holes $885 \mathrm{~A}$ and $886 \mathrm{~B}$. The first is plate motion, which will influence the source contribution to the sediments. Based on the paleopole reconstruction of Sager and Pringle (1988), Holes 885A and 886B were located only $3^{\circ}$ south of the present location at $45^{\circ} \mathrm{N}$ during the late Miocene deposition of sediments at the Unit II/III boundary (R. Larson, pers. comm., 1994), so plate motion should not complicate the interpretation of the eolian sediments above this interval. However, the basement at Sites 885 and 886 has been dated at $\sim 80 \mathrm{Ma}$ (Keller et al., this volume). Paleomagnetic plate rotations indicate that the plate was formed at about $16^{\circ} \mathrm{N}$. Thus, we need to control for plate motion when interpreting the Unit III sediments. Low latitudes are influenced by easterly eolian transport from the North American continent. This supports our hypothesis for some supply of andesitic eolian material to the Unit III hydrothermal sediments.

Tectonic activity in the Asian source area, such as uplift of the Himalayan Mountains or Tibetan Plateau, could impact eolian sedimentation proximal to Sites 885 and 886 as a result of climatic forcing. Geological evidence indicates that uplift pulses are episodic (Copeland et al., 1990; Amano and Taira, 1992; Hovan and Rea, 1993), so the response in the sediment record would be abrupt if the uplift-induced climate forcing is linear. Uplift-forced climatic change (Kutzbach et al., 1989; Ruddiman et al., 1989) is hypothesized to result in cooling of northern Asia and drying of the Eurasian interior. The impact on the eolian sediments in the North Pacific would be manifested as an increase in the mechanical weathering proxies such as in chlorite, quartz, illite, and plagioclase at the expense of chemical weathering 


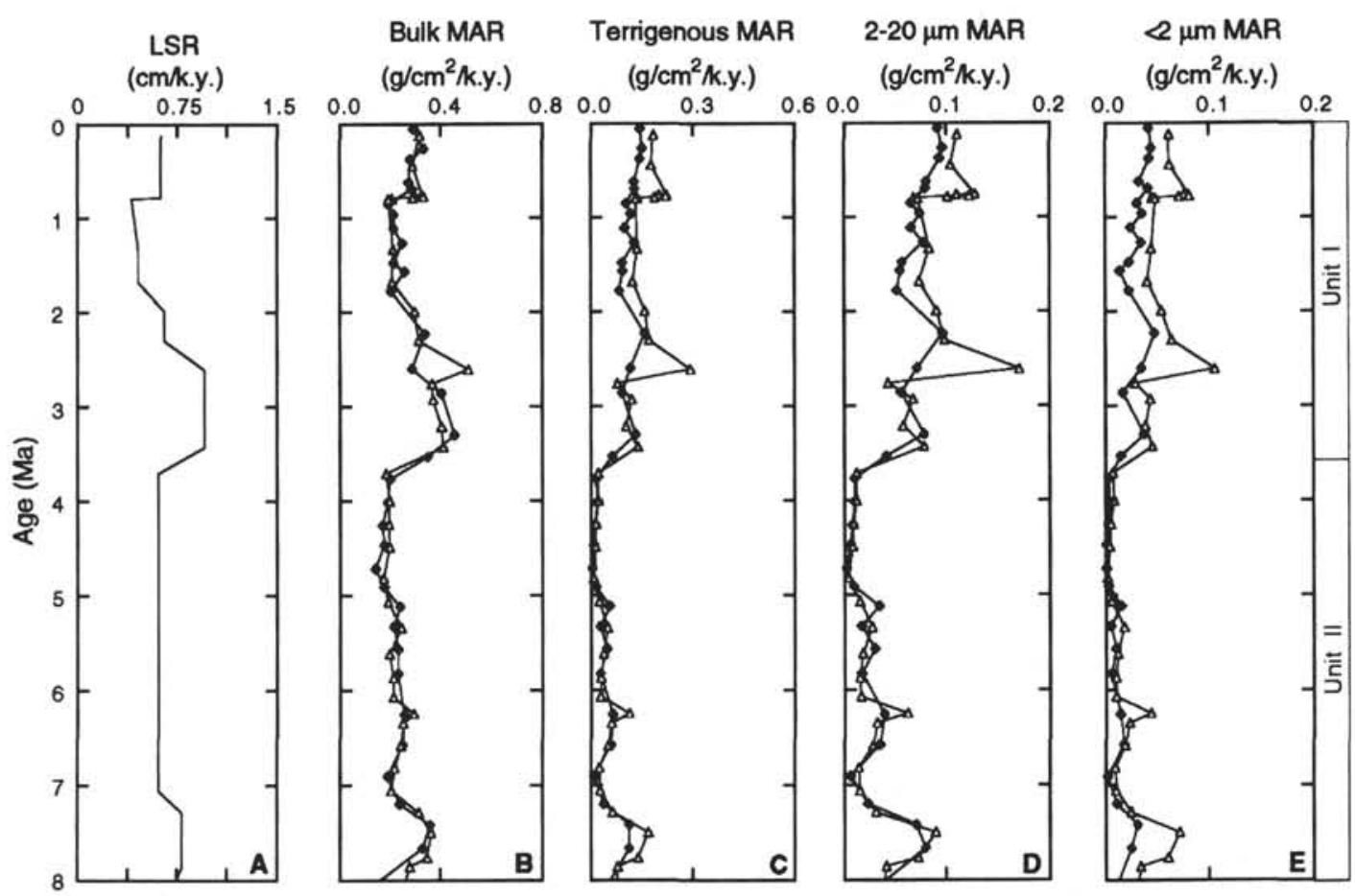

Figure 8. Mass accumulation rates for different sedimentary components vs. age for Holes 885A and 886B.

proxies such as kaolinite and smectite. This climatic change would also produce an increase in terrigenous MARs, as particle production increased because of source region aridification. At Holes $885 \mathrm{~A}$ and $886 \mathrm{~B}$, the shift from a kaolinite-rich mineralogy in the diatom ooze unit to chlorite-rich sediments in the upper red clay unit, concomitant with an increase in the terrigenous MAR are supportive of dramatic environmental changes in Asia during the Pliocene.

The timing of this event in this region of the North Pacific is at about 3.8 Ma. Studies of present-day eolian transport identify the deserts of interior Asia as the source region for the loess deposits in China, as well as the North Pacific eolian sediments (Merrill et al., 1985, 1989). A study by Ding et al. (1992) reports the onset of major loess deposition in China at $2.5 \mathrm{Ma}$. These authors state that the eolian source areas began drying out during the mid to late Pliocene because of plateau uplift. This assertion is supported by the mineralogy in the North Pacific sediments; specifically, the monotonic decline in the concentration of kaolinite in the $2-20 \mu \mathrm{m}$ fraction of the late Miocene to early Pliocene Unit II sediments.

Although the eolian source regions developed in the late Pliocene, the meteorological conditions were not favorable for deposition on the loess plateau at that time. The mechanism invoked by Ding et al. (1992) for the lack of loess deposition during the late Pliocene involves atmospheric circulation changes, inferred from the model of Kutzbach et al. (1989). The authors state that the $500 \mathrm{mb}$ quasistationary trough presently located to the east of the loess plateaus of China was not as well developed during the late Pliocene as it is presently because the Tibetan Plateau elevation was not as high as it is today. Thus, eolian transport from the desert regions was mainly west to east, as opposed to the present northwesterly flow, and the air-mass subsidence over the loess plateau, responsible for dust deposition there, was reduced compared with the present. These meteorological conditions would not preclude deposition of eolian material in the North Pacific; perhaps they even provided a more direct path between the deserts and the ocean than at the present time. The age offset between the onset of loess deposition in China at $2.5 \mathrm{Ma}$ and the sudden increase in eolian deposition in the North Pacific at $3.8 \mathrm{Ma}$ is consistent with the scenario proposed by these authors. The three small peaks in the MARs of the six mineral species in the lower part

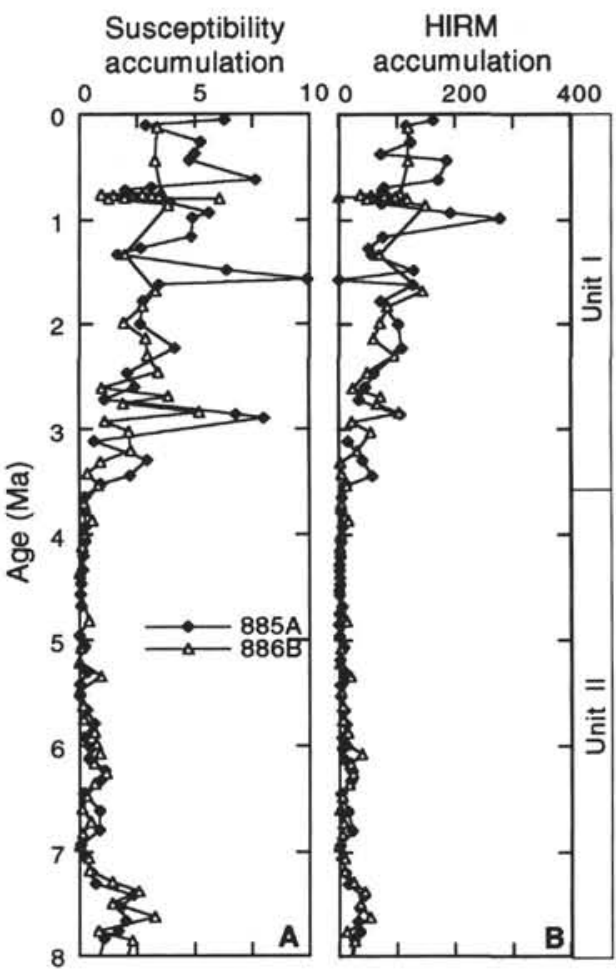

Figure 9. Accumulation rate of magnetic susceptibility and HIRM vs. age for Holes $885 \mathrm{~A}$ and $886 \mathrm{~B}$.

of Unit II at about 7.5, 6.5, and 5.1 Ma may also record smaller pulses of Asian aridification in the late Miocene.

\section{Transport and Deposition Processes}

Changes in the eolian transport and sediment deposition (current winnowing) in this region would be characterized by changes in the 

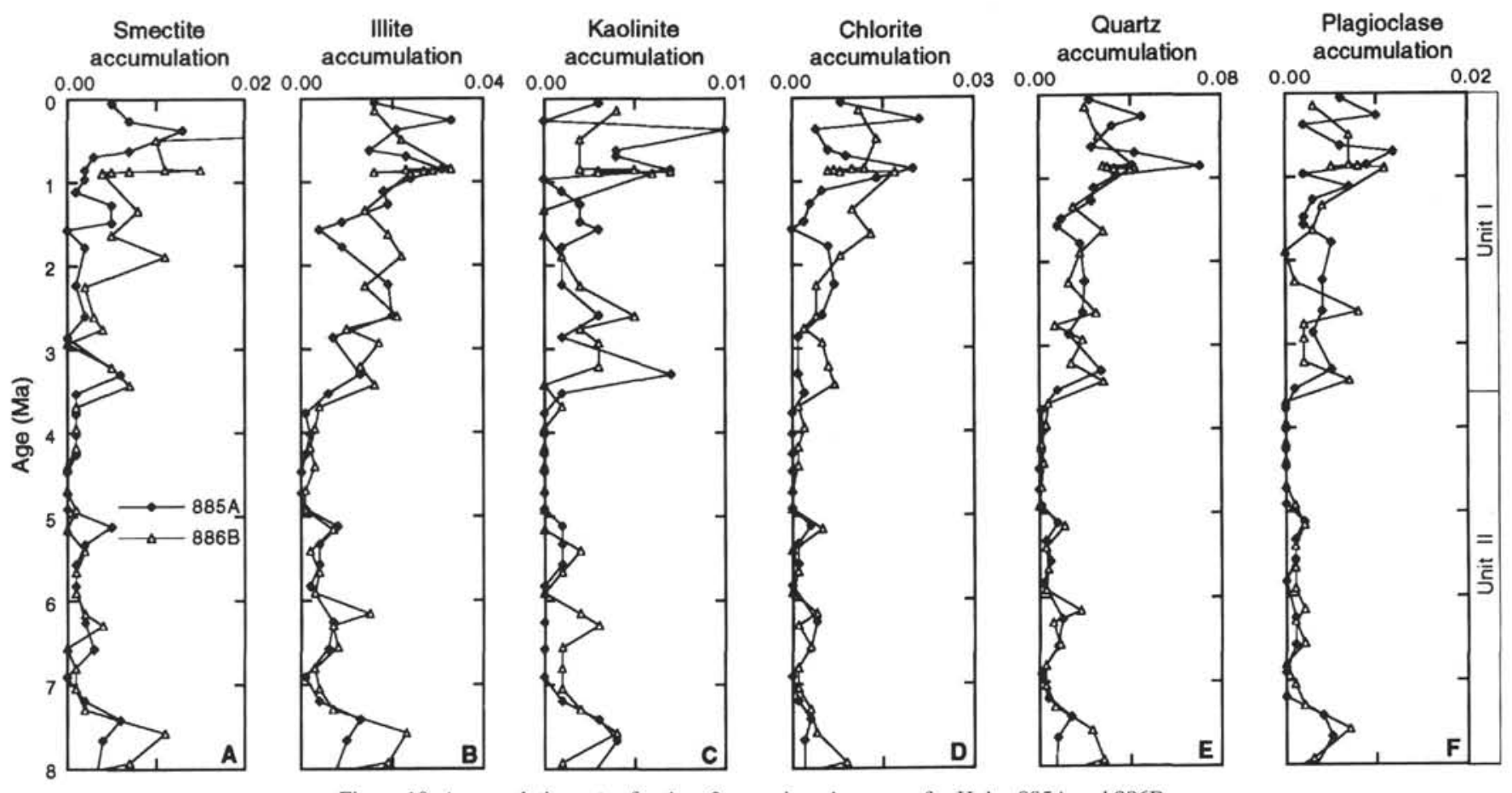

Figure 10. Accumulation rates for the $<2 \mu \mathrm{m}$ minerals vs. age for Holes $885 \mathrm{~A}$ and $886 \mathrm{~B}$.

grain size and changes in the LSRs not accounted for by changing source strengths. Magnetic-iron oxide grain sizes increase at the base of Unit I, and continue to increase throughout the unit. The increase can be explained by increasing transport speed of atmospheric circulation, caused by an increase in the pole-to-equator temperature gradient induced by glaciation. Changes in LSR are generally accounted for by the varying strengths of the terrigenous and biogenic accumulation in these cores. There may be hiatuses in lower clay Unit III, but we do not have the sample resolution in this interval to discern this activity.

\section{Diagenetic Processes}

Another prominent feature of the sediments at Sites 885 and 886 is the sharp signal in the rock magnetic parameters $S$ and $X_{\text {arm }} / X_{\text {If }}$ coupled with the sharp change in mineralogy at $30 \mathrm{mcd}(\sim 4.7 \mathrm{Ma})$. This signal appears in the middle of the diatom ooze unit, during the period with the lowest accumulation of terrigenous sediments in the entire sediment column. The $S$-ratio and $X_{a r m} / X_{l f}$ changes could be the result of diagenetic alteration of the magnetic signal. Iron oxides are soluble under reducing conditions, and the finest grains will be preferentially dissolved over coarser magnetic particles. The low $X_{a r m} / X_{l f}$ ratio between 30 and 34 mcd may be the result of such a grain-size modification. Furthermore, the sharp $X_{a r m} / X_{l f}$ peak at 28-30 mcd could be caused by single-domain magnetite produced by magnetotactic bacteria active at the redox boundary in the sediment at the time of reduction diagenesis. This hypothesis is corroborated by the low S-ratio in this interval. Fine magnetite is more susceptible to dissolution than hematite, and this selective dissolution has altered the bulk magnetic composition in this interval, reducing the proportion of low-coercivity material. The total organic carbon shows a small peak at this depth (Hole 885A, maximum $=0.14 \%$, Hole 886B, maximum $=0.25 \%$; Rea, Basov, Janecek, Palmer-Julson, et al., 1993), although the organic carbon concentrations are extremely low (mean TOC $=0.10 \%$ ) everywhere in the core. The mineralogical variation could suggest a change in source supply, but it is difficult to envision a process that would supply such a short and intense burst of unusual clay mineralogy in the middle of the Pacific Ocean, especially with no increase in the terrigenous MAR. Because the terrigenous material is present in such low concentrations, and is accumulating in such small amounts, the miner- als may be especially susceptible to alteration in this interval. The signal here is probably diagenetic in origin, marked by the dissolution of fine X-ray amorphous clay material in a relatively acidic environment and the creation of transitional minerals during clay halmyrosis. Chamley (1989) proposes this explanation for mineralogical variations in sapropel layers in Eastern Mediterranean sediments. Although the organic carbon concentration here is obviously much lower than the sapropels in the Mediterranean, the very small concentrations of terrigenous material may render it more susceptible to modification. The shape of the signal, with a sharp bottom and a gradational top, was also characteristic of the sapropel diagenetic modification.

\section{SUMMARY}

The magnetic and mineralogy signals from the sediments at Sites 885 and 886 record variations in a number of environmental parameters through time. The relative terrigenous, biogenic silica, and hydrothermal source strengths account for most signal variations. There may be some North American eolian terrigenous component in the hydrothermal sediments in Unit III. The terrigenous component in the diatom ooze and the upper red clay is Asian eolian material. The terrigenous source-area climate and tectonic variations cause a change from a mineralogy rich in chemical weathering products to one rich in mechanical weathering products, at the same time that the accumulation rate and grain size of the terrigenous material increase. These changes are consistent with late Pliocene Tibetan Plateau uplift pulses and Asian aridification. Diagenetic processes recorded in the mineralogy and rockmagnetics indicate that sediments in this region of the North Pacific may have been suboxic to anoxic sometime in the early Pliocene.

\section{ACKNOWLEDGMENTS}

Eve Arnold was supported by a JOI fellowship for this research. This study benefited from the kind assistance of the ODP staff and SEDCO crew aboard the JOIDES Resolution. Ms. Bonnie Wolfe assisted in the processing of the sediments. Jan Bloemendal made helpful suggestions on an early draft of the manuscript. Steve Hovan and Suzanne O'Connell reviewed the manuscript. We thank these individuals for their contributions. 


\section{REFERENCES}

Amano, K., and Taira, A., 1992. Two-phase uplift of the higher Himalayas since 17 Ma. Geology, 20:391-394.

Blank, M., Leinen, M., and Prospero, J.M., 1985. Major eolian inputs indicated by the mineralogy of aerosols and sediments in the western North Pacific. Nature, 314:84-86.

Chamley, H., 1989. Clay Sedimentology: Heidelberg (Springer-Verlag).

Copeland, P., and Harrison, T.M., 1990. Episodic rapid uplift in the Himalaya revealed by ${ }^{40} \mathrm{Ar} /{ }^{39} \mathrm{Ar}$ analysis of detrital $\mathrm{K}$-feldspar and muscovite, Bengal Fan. Geology, 18:354-357.

Ding, Z., Rutter, N., Jingtai, H., and Tungsheng, L., 1992. A coupled environmental system formed at about $2.5 \mathrm{Ma}$ in east Asia. Palaeogeogr., Palaeoclimatol., Palaeoecol., 94:223-242.

Doh, S.-J., King, J.W., and Leinen, M., 1988. A rock-magnetic study of giant piston core LL44-GPC3 from the central North Pacific and its paleoceanographic implications. Paleoceanography, 3:89-111.

Griffin, J.J., Windom, H., and Goldberg, E.D., 1968. The distribution of clay minerals in the World Ocean. Deep-Sea Res. Part A, 15:433-459.

Hovan, S., and Rea, D.K., 1992. The Cenozoic record of continental mineral deposition on Broken and Ninetyeast Ridges, Indian Ocean: southern African aridity and sediment delivery from the Himalayas. Paleoceanography, 7:833-860.

King, J.W., Bloemendal, J., and Gangemi, P., 1989. Paleomagnetic and rockmagnetic stratigraphy of ESOPE core 63, Southern Nares Abyssal Plain. In Schuttenhelm, R.T.E., Auffret, G.A., Buckley, D.E., Cranston, R.E., Murray, C.N., Shepard, L.E., and Spijkstra, A.E. (Eds.), The ESOPE International Expedition, Geoscience Investigations of Two North Atlantic Abyssal Plains: Luxembourg (Office for Official Publications of the European Community), 611-636.

Kutzbach, J.E., Guetter, P.J., Ruddiman, W.F., and Prell, W.L., 1989. Sensitivity of climate to late Cenozoic uplift in southern Asia and the American west: numerical experiments. J. Geophys. Res., 94:18393-18407.

Kyte, F.T., Leinen, M., Heath, G.R., and Zhou, L., 1993. Cenozoic sedimentation history of the central North Pacific: inferences from the elemental geochemistry of Core LL44-GPC3. Geochim. Cosmochim. Acta, 57:1719-1740.

Landa, E.R., and Gast, R.G., 1973. Evaluation of crystallinity in hydrated ferric oxides. Clays Clay Miner., 21:121-130.

Leinen, M., 1985. Quartz content of Northwest Pacific Hole 576A and implications for Cenozoic eolian transport. In Heath, G.R., Burckle, L.H., et al., Init. Repts. DSDP, 86: Washington (U.S. Govt. Printing Office), 581-588.
1989. The late Quaternary record of atmospheric transport to the Northwest Pacific from Asia. In Leinen, M., and Sarnthein, M. (Eds.), Paleoclimatology and Paleometeorology: Modern and Past Patterns of Global Atmospheric Transport: New York (Kluwer), 693-732.

Merrill, J.T., Bleck, R., and Avila, L., 1985. Modeling transport to the Marshall Islands. J. Geophys. Res., 90:12927-12936.

Merrill, J.T., Uematsu, M., and Bleck, R., 1989. Meteorological analysis of long range transport of mineral aerosols over the North Pacific. J. Geophys. Res., 94:8584-8598.

Oldfield, F., Hunt, A., Jones, D.H., Chester, R., Dearing, J.A., Olsson, L., and Prospero, J.M., 1985. Magnetic differentiation of atmospheric dusts. Nature, 317:516-518.

Rea, D.K., Basov, I.A., Janecek, T.R., Palmer-Julson, A., et al., 1993. Proc. $O D P$, Init. Repts., 145: College Station, TX (Ocean Drilling Program).

Robinson, S.G., 1986. The late Pleistocene paleoclimatic record of North Atlantic deep-sea sediments revealed by mineral-magnetic measurements. Phys. Earth Planet. Inter., 42:22-47.

Ruddiman, W.F., Prell, W.L., and Raymo, M.E., 1989. Late Cenozoic uplift in southern Asia and the American West: rationale for general circulation modeling experiments. J. Geophys. Res., 94:18379-18391.

Sager, W.W., and Pringle, M.S., 1988. Mid-Cretaceous to early Tertiary apparent polar wander path of the Pacific Plate. J. Geophys. Res., 93:11753-11771.

Schramm, C.T., 1989. Cenozoic climatic variation recorded by quartz and clay minerals in North Pacific Sediments. In Leinen, M., and Sarnthein, M. (Eds.), Paleoclimatology and Paleometeorology: Modern and Past Patterns of Global Atmospheric Transport: Dordrecht (NATO ASI Series), 805-839.

- Abbreviations for names of organizations and publications in ODP reference lists follow the style given in Chemical Abstracts Service Source Index (published by American Chemical Society),

Date of initial receipt: 4 April 1994

Date of acceptance: 1 August 1994

Ms 145SR-113 